Revised MSSP Manuscript (15-748R1) Submitted in October 2015

\title{
Harmonic Amplitude Dependent Dynamic Stiffness of Hydraulic Bushings: Alternate Nonlinear Models and Experimental Validation
}

\author{
Luke Fredette, Jason T. Dreyer, Todd E. Rook, Rajendra Singh \\ Acoustics and Dynamics Laboratory, Smart Vehicle Concepts Center, \\ Department of Mechanical and Aerospace Engineering, \\ The Ohio State University, Columbus, OH 43210, USA \\ "Corresponding Author: E-mail: singh.3@,osu.edu, Phone: +1-614-292-9044
}

\begin{abstract}
The dynamic stiffness properties of automotive hydraulic bushings exhibit significant amplitude sensitivity which cannot be captured by linear time-invariant models. Quasi-linear and nonlinear models are therefore proposed with focus on the amplitude sensitivity in magnitude and loss angle spectra (up to $50 \mathrm{~Hz}$ ). Since the model parameters of a production bushing are unknown, dynamic stiffness tests and laboratory experiments are utilized to extract model parameters. Nonlinear compliance and resistance elements are incorporated, including their interactions in order to improve amplitude sensitive predictions. New solution approximations for the new system equations (containing both nonlinear compliance and resistance elements) refine the multi-term harmonic balance term method. Quasi-linear models yield excellent accuracy but lack the ability to predict trends in amplitude sensitivity since they rely on available dynamic stiffness measurements. Nonlinear models containing both nonlinear resistance and compliance elements yield superior predictions to those of prior models (with a single resistance or compliance nonlinearity), while also providing more physical insight. Suggestion for further work is briefly mentioned.
\end{abstract}


Key Words: Hydraulic devices; nonlinear system simulation; multi-term harmonic balance method; vehicle application; sinusoidal testing

\section{Introduction}

Hydraulic elastomeric devices are often employed in automotive powertrain and suspension systems because of their unique dynamic properties, leading to both vibration isolation and motion control [1-18]. These properties are achieved by an internal fluid system working in tandem with the elastomeric structure of a bushing. Despite some similarities with hydraulic engine mounts that have been extensively studied [1-9], the behavior of hydraulic bushings is quite different and merits its own in-depth studies [10-18]. Prior, though limited, investigations of these devices have largely focused on simpler transfer function type formulations based on the linear time-invariant (LTI) system theory [10-16]. For instance, Arzanpour and Golnaraghi [12] developed a reduced order linear system model for a hydraulic bushing which attempts to capture some aspects of the physics, such as fluid resistance, compliance, and inertance. Chai et al [13-16] further developed the linear models for frequency and time domain characteristics for a laboratory bushing device and even introduced a nonlinear fluid resistance term [17]. Fredette et al. [18] recently developed a new laboratory experiment to measure the nonlinear fluid compliance of hydraulic bushing pumping chambers.

Under harmonic excitation, hydraulic bushings exhibit significant amplitude dependent behavior [10, 11, 14-18], which cannot be described by the linear time-invariant system theory. Both the mechanical (rubber path) stiffness and fluid compliance elements of the bushings arise from the molded interfacial elastomeric material. Since most elastomeric materials exhibit inelastic behavior, nonlinear fluid compliance behavior has been suggested, $[4,5,7-9,17,18]$ 
but the amplitude sensitivity has never been mathematically described. The chief goal of this article is therefore to propose new or improved quasi-linear and nonlinear reduced-order hydraulic bushing models, predict amplitude sensitivity characterization under harmonic loading, and experimentally validate alternate nonlinear models. Additionally, the prior work [17] on the multi-term harmonic balance method will be extended and refined to explain the underlying physics.

\section{Problem formulation}

Modeling of hydraulic bushings is challenging due to their complexity that arises due to the interacting nonlinear design features, nonlinear materials, and variation in production bushing designs. Hydraulic bushings are typically constructed of an elastomeric material constrained by a metal inner and outer sleeve, as shown in Figure 1. Fluid-filled internal chambers deform when the bushing is displaced, pumping the fluid through a long passage between the chambers. A Kelvin-Voigt (linear system) model is assumed for the elastomeric structural path, while this article focuses on nonlinear fluid elements.

The lumped parameter modeling method is suitable for the fluid system contained within hydraulic bushings at low frequencies (up to $50 \mathrm{~Hz}$ ), where the corresponding wavelength is much larger than the bushing dimensions. For the example case, model parameters include fluid compliance, $C$, and effective pumping area, $A$, for each chamber, the fluid resistance, $\mathbf{R}_{i}$, and inertance, $I_{i}$, of the single inertia track, and the stiffness, $K_{r}$, and viscous damping coefficient, $c_{r}$, of the rubber structure within the device. The state variables of the system include the absolute pressure, $p$, in each chamber (left $(L)$ and right $(R)$ ) as well as the volume flow rate of fluid between the chambers, $q_{i}$. The outer sleeve is considered to be constrained, so the system is 
excited by the displacement of the inner sleeve, $x(t)$; the force transmitted to the outer sleeve, $F_{T}$, is the response.

Tractable lumped parameter models are needed to yield reasonable predictions of amplitude sensitive dynamic stiffness, which is a useful metric for design and diagnostic purposes, and to provide physical insight. Accordingly, the specific objectives of this article are as follows. (1) Propose and experimentally validate quasi-linear and nonlinear bushing models (with nonlinear resistance and compliance elements) which capture amplitude sensitivity in the example case of a production bushing with a single inertia track as displayed in Figure 1. (2) Refine and utilize the semi-analytical multi-term harmonic balance method (MHBM) to construct dynamic stiffness spectra and gain physical insight into the role and interaction of nonlinearities in the model. The scope of the work is limited to sinusoidal excitation only with peak-to-peak displacement amplitudes of $0.1 \mathrm{~mm}, 0.5 \mathrm{~mm}, 1.0 \mathrm{~mm}$, and $2.0 \mathrm{~mm}$ over a frequency range from 1 to $50 \mathrm{~Hz}$ to capture the amplitude sensitivity of the tuned dynamic properties.

The bushing is characterized by a cross-point dynamic stiffness, assuming a sinusoidal excitation at angular frequency $\Omega$, where $x_{m}$ is the mean component and $x_{a}$ is the peak-to-peak excitation amplitude,

$$
x(t)=x_{m}+\frac{x_{a}}{2} \sin (\Omega t) .
$$

The force transmitted to a rigid base is calculated by summing the contributions from parallel structural and fluid paths. The force through the elastomeric structural path may be directly 
calculated, $F_{r}=K_{r} x+c_{r} \dot{x}$, while the force through the fluid path requires a solution to the following governing fluid system equations,

$$
\begin{gathered}
C_{L} \dot{p}_{L}=A_{L} \dot{x}-q_{i}, \\
C_{R} \dot{p}_{R}=-A_{R} \dot{x}+q_{i}, \\
I_{i} \dot{q}_{i}=p_{L}-p_{R}-\mathbf{R}_{i} q_{i} .
\end{gathered}
$$

Note that both pressure $p_{L}$ and $p_{R}$ time histories are needed for the calculation, since the fluid path force is: $F_{f}=A_{L} p_{L}-A_{R} p_{R}$. The equations can be expressed in the following canonical state form allowing direct calculation of the total transmitted force from the fluid state; here, $\underline{u}=\left[\begin{array}{ll}x & \dot{x}\end{array}\right]^{T}$ is the excitation vector and $\underline{z}$ is the fluid state vector,

$$
\begin{gathered}
\underline{z}=\left\{\begin{array}{l}
p_{L} \\
p_{R} \\
q_{i}
\end{array}\right\}, \\
\underline{\dot{z}}=\left[\begin{array}{ccc}
0 & 0 & -\frac{1}{C_{L}} \\
0 & 0 & \frac{1}{C_{L}} \\
\frac{1}{I_{i}} & -\frac{1}{I_{i}} & -\frac{\mathbf{R}_{i}}{I_{i}}
\end{array}\right] \underline{z}+\left[\begin{array}{cc}
0 & \frac{A_{L}}{C_{L}} \\
0 & -\frac{A_{R}}{C_{R}} \\
0 & 0
\end{array}\right] \underline{u}, \\
F_{T}=\left[\begin{array}{lll}
A_{L} & -A_{R} & 0
\end{array}\right] \underline{z}+\left[\begin{array}{ll}
K_{r} & c_{r}
\end{array}\right] \underline{u} .
\end{gathered}
$$


When the system is linear time-invariant (as shown in Figure 1), the total transmitted force will be sinusoidal where $F_{m}$ is the mean component, $F_{a}$ is the peak-to-peak force amplitude, and $\phi$ is the phase (or loss angle),

$$
F_{T}(t)=F_{m}+\frac{F_{a}}{2} \sin (\Omega t-\phi)
$$

In this case, the harmonic excitation and response allows for a precise definition of complexvalued dynamic stiffness, $\tilde{K}$, at $\Omega$, given by magnitude and angle respectively,

$$
\begin{aligned}
& |\tilde{K}(\Omega)|=\left.\frac{F_{a}}{x_{a}}\right|_{\Omega}, \\
& \angle \tilde{K}(\Omega)=\phi(\quad) .
\end{aligned}
$$

\section{Development of quasi-linear model}

\subsection{Linear time-invariant formulation}

A transfer function representation of dynamic stiffness is easily obtained by applying the Laplace Transform to the differential equations which govern the fluid system. This allows for an algebraic solution of the pressures, $p_{L}$ and $p_{R}$ in Laplace $(s)$ domain, and thus a direct calculation of the dynamic stiffness transfer function (in $s$ domain) as,

$$
K(s)=\frac{\alpha_{3} s^{3}+\alpha_{2} s^{2}+\alpha_{1} s+\alpha_{0}}{s^{2}+\alpha_{5} s+\alpha_{4}},
$$

where the coefficients are defined as follows,

$$
\alpha_{0}=\frac{K_{r}}{I_{i}}\left(\frac{1}{C_{L}}+\frac{1}{C_{R}}\right)+\frac{1}{I_{i}} \frac{\left(A_{L}-A_{R}\right)^{2}}{C_{L} C_{R}}
$$




$$
\begin{gathered}
\alpha_{1}=K_{r} \frac{\mathbf{R}_{i}}{I_{i}}+c_{r} \frac{1}{I_{i}}\left(\frac{1}{C_{L}}+\frac{1}{C_{R}}\right)+\frac{\mathbf{R}_{i}}{I_{i}}\left(\frac{A_{L}^{2}}{C_{L}}+\frac{A_{R}^{2}}{C_{R}}\right) \\
\left.\alpha_{2}=K_{r}+c_{r} \frac{\mathbf{R}_{i}}{I_{i}}+\left(\frac{A_{L}^{2}}{C_{L}}+\frac{A_{R}^{2}}{C_{R}}\right)\right\} \\
\alpha_{3}=c_{r}, \\
\left.\alpha_{4}=\frac{1}{I_{i}} \frac{(1}{\left(C_{L}\right.}+\frac{1}{C_{R}}\right), \\
\alpha_{5}=\frac{\mathbf{R}_{i}}{I_{i}} .
\end{gathered}
$$

Unlike the transfer function method based on the LTI system theory, significant amplitude dependence is observed in the measured dynamic stiffness $\tilde{K}_{d}$, of a production

bushing. It is displayed in Figure 2 for both $\left|\tilde{K}_{d}\right|$ and $\phi$. Here, frequency is normalized by the frequency at which the loss angle is maximum, $\hat{\Omega}=\quad /_{b}$, and the dynamic stiffness magnitude is normalized by the static stiffness, $\left|\hat{K}_{d}\right|=\left|\tilde{K}_{d}\right| / K_{s}$.

\subsection{Quasi-linear formulation (Model I)}

Since the LTI model is not capable of capturing the effects in Figure 2, an extension to a quasi-linear model is necessary. Allowing model coefficients $\alpha_{0}, \alpha_{1}, \alpha_{2}, \alpha_{3}, \alpha_{4}$, and $\alpha_{5}$ or physical parameters $I_{i}, R_{i}, C, A, K_{r}$, and $c_{r}$ to be influenced by the state variables of the fluid system may better characterize the physical behavior observed in hydraulic bushings [2] and thus capture the amplitude dependent effects. The parameters most sensitive to changes in $x_{a}$ are 
identified by an empirical parameter estimation method. This is accomplished by approximating $\tilde{K}_{d}\left(\Omega, x_{a}\right)$ for a production bushing (such as the results of Figure 2) with an assumed form,

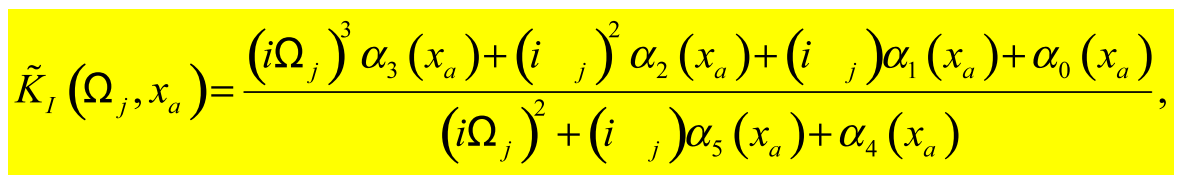

where $s \rightarrow i \Omega$ from equation (7a). A nonlinear constrained optimization with an interior-point algorithm [21] is used to optimize the $\alpha$ coefficients to minimize the difference between the measured $\tilde{K}_{d}$ and the quasi-linear model, $\tilde{K}_{I}$, at a given $x_{a}$. The optimization is achieved with the numerator and denominator $\alpha$ coefficients instead of the physical parameters $\left(I_{i}, R_{i}, C, A\right.$, $K_{r}$, and $c_{r}$ ) because of the numerical difficulties resulting from an extreme dynamic range displayed by the physical parameters. An objective function $\left(\varepsilon_{\alpha}\right)$ is defined as follows, where $\tilde{K}_{d}\left(\Omega_{j}, x_{a}\right)$ is the measured dynamic stiffness of the production bushing at a given amplitude $x_{a}$ which has been measured at frequency, $\Omega_{j}$,

$$
\varepsilon_{\alpha}\left(x_{a}\right)=\sum_{j}\left(\tilde{K}_{I}\left(\Omega_{j}, x_{a}\right)-\tilde{K}_{d}\left({ }_{j}, x_{a}\right) \|^{2}\right)
$$

Physical parameters may be calculated from the optimized values of $\alpha_{j}$ using equations $(7 b-g)$. Since the bushing is geometrically symmetric (barring any manufacturing variations), the assumptions $C_{L}=C_{R}=C$ and $A_{L}=A_{R}=A$ reasonably reduce the number of physical parameters. With these assumptions, the number of physical parameters reduces to six, which is equal to the number of transfer function coefficients. However, the set of equations is not linearly independent. An assumed value for fluid inertance (based on geometry and density 
calculations) allows for the direct estimation of the other parameters, $K_{r}, c_{r}, \mathbf{R}_{i}, C$, from equations $(7 \mathrm{~b}-7 \mathrm{~g})$ :

$$
\begin{gathered}
K_{r}=\frac{\alpha_{0}}{\alpha_{4}} \\
c_{r}=\alpha_{3}, \\
\mathbf{R}_{i}=\alpha_{5} I_{i}, \\
C=\frac{2}{\alpha_{4} I_{i}} .
\end{gathered}
$$

This leaves two equations with a single unknown, $A$. Running yet another (though similar) optimization using pumping area $A$ (assuming symmetry) as the only parameter reveals that the effective value is equal to the one calculated from equation (7d),

$$
A=\frac{C}{2}\left(\alpha_{2}-K_{r}-\frac{c_{r} \mathbf{R}_{i}}{I_{i}}\right)
$$

The coefficients $\alpha_{j}$ are then recalculated from the new physical parameters, producing the empirical $\tilde{K}_{I}$. The results of this process for each measured amplitude are plotted in Figures 3 and 4 showing reasonable accuracy in dynamic stiffness amplitude and loss angle spectra, respectively. The identified parameters are compared in Table 1, where each parameter is normalized by its value with a $0.1 \mathrm{~mm}$ amplitude peak-to-peak excitation, such that $\hat{\mathbf{R}_{i, j}}=\mathbf{R}_{i, j} / \mathbf{R}_{i, 1}$, etc. The approximation error of this model will be quantified later. 
These results suggest that many of the model parameters are amplitude sensitive. Quasilinear modeling is a reasonable method of estimating the amplitude sensitivity, but it requires sinusoidal measurements on a physical device at each amplitude of interest and offers no physical insight.

\section{Development of nonlinear models}

\subsection{Nonlinear resistance element (Model II)}

Nonlinear models take a different mathematical approach than a quasi-linear model, basing the governing parameters directly on theory (in terms of differential equations) with some augmentation from limited laboratory experiment(s) rather than empirical curve-fitting of dynamic stiffness. The mathematical formulation of a nonlinear element may then implicitly introduce amplitude sensitivity to the model. For instance, in previous work [17], a nonlinear fluid resistance element was introduced to the fluid system to produce amplitude dependent damping. This element, which is also used in the current article, is based on a turbulent flow formulation [22],

$$
\mathbf{R}_{i}\left(q_{i}\right)=\frac{0.242 l_{i} \mu^{0.25} \rho^{0.75}}{D_{i}^{4.75}}\left|q_{i}\right|^{0.75}=\beta_{i}\left|q_{i}\right|^{0.75}
$$

where $D_{i}$ and $l_{i}$ are the hydraulic diameter and length of the inertia track respectively, $\mu$ and $\rho$ are the dynamic viscosity and density of the pumping fluid respectively, and $q_{i}$ is the volume flow rate through the track. Thus, $\beta_{i}$ quantifies the effect of fluid properties and flow passage geometry on $\mathbf{R}_{i}$; typical values (in consistent units) from this study are on the order of $10^{12}$. The large value is due to the small diameter of the flow passage. Some amplitude sensitivity can be 
achieved here, since the amplitude of $q_{i}$ increases with $x_{a}$. However, significant error still exists, which will be quantified later and compared with other models. To improve amplitude sensitivity characterization, an additional nonlinear element is needed.

\subsection{Nonlinear compliance element (Model III)}

Compliance in the fluid system results from several sources, including the compressibility of the pumping fluid, the much larger compressibility of any small amounts of entrained gas in the fluid, and the flexibility of the chamber walls (denoted by the subscript $c$ ) [23]. Assuming there is a very small amount of gas and the fluid is nearly incompressible, the chamber compliance is much higher than the other sources, so the compliance may be simplified as, $C \approx C_{c}$. Chamber compliance is related to deformation of the elastomeric chamber volume $V_{c}$ under pressure, $p_{c}$.

The pumping volume for each chamber ( $L, R$, as shown in Figure 1) depends on the displacement of the inner sleeve and the pressure in the chamber, $V_{c}=V_{c}\left(p_{c}, x\right)$. Some coupling is also present, wherein a non-zero static displacement alters the pressure dependence, and the pressure in the chamber affects the volume vs. displacement relation. These unknown interdependencies are simplified by introducing the compliance $(C)$ and effective pumping area $(A)$ parameters. Each parameter is related to the volume by a linearization of the volume function about an operating point as follows,

$$
C(p)=\left.\frac{\partial V}{\partial p}\right|_{x=0},
$$




$$
A=\left.\frac{\partial V}{\partial x}\right|_{p=0}
$$

where $p$ is the mean pressure in each chamber. In order to experimentally obtain the nonlinear compliance parameter, the relation between volume and pressure must be measured.

In prior work, Kim et al. [5] characterized the compliance of hydraulic mounts by filling the pumping chambers with water and attaching a graduated column to the fill port so that the height of the fluid column may be measured. The gas above the fluid column is then pressurized so that both the volume and the pressure in the fluid chambers can be measured. If any gas is left inside the chambers (which is difficult to verify) then the additional compliance of that gas introduces error into the measurement. To resolve this difficulty, Fredette et. al. [18] proposed a new experiment to measure the fluid compliance of hydraulic bushing pumping chambers using pressurized air so that only a single fluid is present in the chambers. Finite element models are utilized in other studies [7-9, 17] to computationally determine the fluid compliance. Confidence in the computational result relies on accurate characterization of material properties, which is difficult to verify for the elastomeric materials used in production bushings.

Using the current author's experiment [18] with the production bushing yields the $V_{c}\left(p_{c}\right)$ relation of the pumping chambers, illustrated in Figure 5. A least-squares curve fit is applied, leading to the following polynomial form, where $\beta_{c}$ are empirical parameters,

$$
V_{c}\left(p_{c}\right)=\frac{\beta_{c, 3}}{3} p_{c}^{3}+\frac{\beta_{c, 2}}{2} p_{c}^{2}+\beta_{c, 1} p_{c}+\beta_{c, 0}
$$

The compliance of the chambers is calculated from equations (12) and (14) as, 


$$
C_{c}\left(p_{c}\right)=\beta_{c, 3} p_{c}^{2}+\beta_{c, 2} p_{c}^{2}+\beta_{c, 1}
$$

Although the above mentioned cubic polynomial is chosen to fit the $V_{c}\left(p_{c}\right)$ measurements, curve-fitting with other functional forms is certainly possible. However, chamber pressures under dynamic loading (particularly at high excitation amplitudes) exceed the measured pressure range of the compliance characterization experiment [18]. Therefore, the compliance curve must be extrapolated beyond the measured regime during simulation. Quadratic, logarithmic, and power functions have been investigated as alternatives, but the cubic model of equation (14) yields the greatest accuracy in dynamic stiffness predictions.

The compliance measured by this experiment describes the two chambers of the bushing acting together. The parameters of Figure $1\left(C_{L}\right.$ and $\left.C_{R}\right)$ describe the compliance of only a single chamber. Since the inertia track is much stiffer than the pumping chambers, the effective fluid compliance parameter is given by $C_{L}=C_{R}=0.5 \sigma_{C} C_{c}\left(p_{c}\right)$. Here, $\sigma_{C}$ is an empirical correction factor which accounts for the dynamic stiffness amplification of the elastomeric structural material (under harmonic loading since the compliance is measured under static conditions). The correction factor also resolves any tuning error resulting from inaccurate fluid inertance estimation, given the complex flow passages of the inertia track. The correction factor is calculated by comparing the peak frequency in the dynamic stiffness loss angle spectrum from a $0.1 \mathrm{~mm}$ amplitude measurement with the simulated peak frequency using the nonlinear compliance with $\sigma_{C}=1$. The peaks are brought together by supplying a new value of $\sigma_{C}$, which is roughly equivalent to the square of the ratio of simulated to measured peak frequencies. A typical correction factor value from this study is found to be on the order of 0.1 . While this value 
is relatively low, further improvement requires additional studies focused on the many

experimental and physical considerations which affect it; this is beyond the scope of this article.

This nonlinear compliance is normalized by the value of the $0.1 \mathrm{~mm}$ amplitude quasilinear compliance parameter and then plotted against this parameter in Figure 6. It is clear from this plot that the nonlinear compliance will introduce further amplitude sensitivity into the model, since the magnitude of chamber pressures increases with excitation amplitude, $x_{a}$.

\subsection{Nonlinear resistance and compliance elements used concurrently (Model IV)}

Inclusion of both the nonlinear R and C elements (from Models II and III, respectively) should incorporate interactions between nonlinearities and thus generate additional amplitude sensitivity. Comparison of these models requires a solution method, discussed in the next section. The model error will be quantified later.

\section{Numerical solution of nonlinear models (II, III, and IV)}

Unlike the frequency-domain representation of dynamic stiffness which assumes an explicit form in the quasi-linear model, a numerical solution is needed for models containing nonlinear elements. The governing ordinary differential equations are solved by assuming a set of initial condition given computational parameters like time steps and tolerances. The timedomain solution is generated using a Runge-Kutta integrator [21]. The early part of solution is dominated by the initial conditions, but the response rapidly converges to steady-state given significant damping in the system. The final period of the simulated steady-state response is used to calculate the dynamic stiffness as follows. 
Since models II, III, and IV include nonlinear elements, the transmitted force can no longer be assumed to be purely sinusoidal and some harmonic distortion will occur. In this case, a sinusoidal approximation may be curve-fit to the transmitted force, providing the basis for an effective dynamic stiffness [24]. A constrained nonlinear optimization with an interior-point algorithm [21] is utilized to calculate the force amplitude, $F_{a}$, and phase shift, $\phi$, which minimize the approximation error $\varepsilon_{F}$, defined as follows,

$$
\mathcal{\varepsilon}_{F}=\sum_{j=1}^{M}\left\{F_{T, j}-F_{m}-\frac{F_{a}}{2} \sin \left(\Omega t_{j}-\phi\right)\right)
$$

where $M$ is the number of data points in one period of the simulated $F_{T}$ time series excited at frequency, $\Omega$. The mean force component is calculated and subtracted prior to the optimization.

Figure 7 shows the predictions of model IV, showing similar amplitude dependence trends as the measured stiffness spectra. Figures 8 and 9 compare the simulated dynamic stiffness magnitudes and loss angles of models II, III, and IV with measured spectra from the production bushing. The agreement between measurement and the nonlinear models is not as close as it is with the quasi-linear model (with amplitude dependent parameters). Observe that neither $\tilde{K}_{I I}$ nor $\tilde{K}_{I I I}$ fully capture the amplitude sensitivity of $\tilde{K}_{d}$ but the interaction between the two nonlinearities in model IV improves the approximation, though significant error still exists, particularly for low excitation amplitudes. Some of the error may be due to excessive extrapolation of the compliance relationship. Measurement of pressures up to about $200 \mathrm{kPa}$ are considered due to experimental limitations [18]; however, dynamic pressures up to $800 \mathrm{kPa}$ are found in simulation. Nevertheless, the amplitude dependent accuracy of this model (despite the 
extrapolation) indicates that the proposed model (IV) is satisfactory since it captures much of the underlying physics.

\section{Construction of semi-analytical solution using the multi-term harmonic balance method}

Unlike the numerical integration method, more insight may be gained by using the multiterm harmonic balance method (MHBM) [17, 21, 22] to generate a semi-analytical solution for model IV. To begin, time $(t)$ is scaled by the excitation frequency, $\theta=\Omega t$, where $\theta$ is the scaled time and $\Omega$ is the excitation frequency in $\mathrm{rad} / \mathrm{s}$. The time derivative is related to the scaled time derivative, $\dot{x}=\Omega x^{\prime}$, where the dot notation refers to derivative with respect to time, and the prime notation refers to derivation with respect to scaled time. The state variables are also scaled as,

$$
\begin{aligned}
& p_{L}=p_{b} \hat{p}_{L}, \\
& p_{R}=p_{b} \hat{p}_{R}, \\
& q_{i}=q_{b} \hat{q}_{i} .
\end{aligned}
$$

Here, the subscript $b$ denotes a baseline or reference value, and the over-hat symbol denotes normalization. The system state vector $\underline{z}$ is normalized, where $\underline{\underline{S}}_{z}$ is the magnitude scaling matrix,

$$
\underline{z}=\underline{\underline{S}}_{z} \underline{\underline{z}}=\left[\begin{array}{ccc}
p_{b} & 0 & 0 \\
0 & p_{b} & 0 \\
0 & 0 & q_{b}
\end{array}\right]\left\{\begin{array}{l}
\hat{p}_{L} \\
\hat{p}_{R} \\
\hat{q}_{i}
\end{array}\right\}
$$

When a linear compliance parameter along with a nonlinear resistance term is used, the system equations are effectively reduced into the following single governing equation as utilized previously by Chai et al. [17]: 


$$
I_{i} \ddot{q}_{i}+\mathbf{R}_{i}\left(q_{i}\right) \dot{q}_{i}+\left(\frac{1}{C_{L}}+\frac{1}{C_{R}}\right) q_{i}=\left(\frac{A_{L}}{C_{L}}+\frac{A_{R}}{C_{R}}\right) \dot{x}
$$

where $\mathbf{R}_{i}\left(q_{i}\right)$ is a nonlinear parameter. A semi-analytical solution approximation was then generated for $q_{i}(t)$ by Chai et al. [17] based on equation (19). Conversely, when the nonlinear compliance and nonlinear resistance elements are concurrently employed, the above mentioned reduction to a single equation is no longer possible. The MHBM in procedure of prior work [17, 21] must therefore be modified to work with a system of equations containing nonlinear compliance parameters. The system equations are converted from the form of equation (4) into residual $(\underline{\gamma})$ form,

$$
\underline{\gamma}=\left\{\begin{array}{cc}
C\left(p_{L}\right) \Omega p_{L}^{\prime}+q_{i}-A_{L} & x^{\prime} \\
C\left(p_{R}\right) \Omega p_{R}^{\prime}-q_{i}+A_{R} & x^{\prime} \\
I_{i} \Omega q_{i}^{\prime}+\mathbf{R}_{i}\left(q_{i}\right) q_{i}+p_{R}-p_{L}
\end{array}\right\},
$$

where $C(p)$ is the experimentally determined compliance function in equation $(15), \mathbf{R}_{i}\left(q_{i}\right)$ is the theoretical fluid resistance in the inertia track based on a turbulent flow formulation. Additionally, $\underline{\gamma}$ is normalized using the residual scaling matrix,

$$
\underline{\gamma}=\underline{\underline{S}}_{\gamma} \underline{\hat{\gamma}}=\left[\begin{array}{ccc}
q_{b} & 0 & 0 \\
0 & q_{b} & 0 \\
0 & 0 & p_{b}
\end{array}\right] \hat{\underline{\gamma}} .
$$

This yields the normalized residual function $\underline{\gamma}(\underline{z})$ in terms of normalized state variables, 


$$
\hat{\underline{\gamma}}=\left\{\begin{array}{c}
C\left(p_{b} \hat{p}_{L}\right) \frac{\Omega p_{b}}{q_{b}} \hat{p}_{L}^{\prime}+\hat{q}_{i}-\frac{A_{L} \Omega}{q_{b}} x^{\prime} \\
C\left(p_{b} \hat{p}_{R}\right) \frac{\Omega p_{b}}{q_{b}} p_{R}^{\prime}-\hat{q}_{i}+\frac{A_{R} \Omega}{q_{b}} x^{\prime} \\
\frac{I_{i} \Omega q_{b}}{p_{b}} \hat{q}_{i}^{\prime}+\frac{q_{b}}{p_{b}} \mathbf{R}_{i}\left(q_{b} \hat{q}_{i}\right) \hat{q}_{i}+\hat{p}_{R}-\hat{p}_{L}
\end{array}\right\}
$$

Application of the harmonic balance method in time-domain is possible, but computationally expensive and inaccurate. This analysis functions better in the frequency domain, for which a Discrete Fourier Transform (DFT) is required [17]. In this case, it is easier to define the inverse DFT matrix,

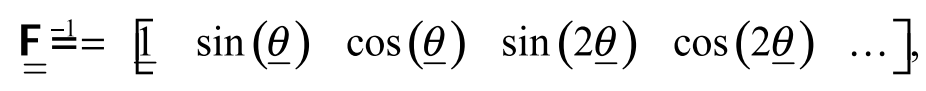

where $\underline{\theta}$ is the normalized time vector. A derivative in time domain corresponds to multiplication by the derivative matrix,

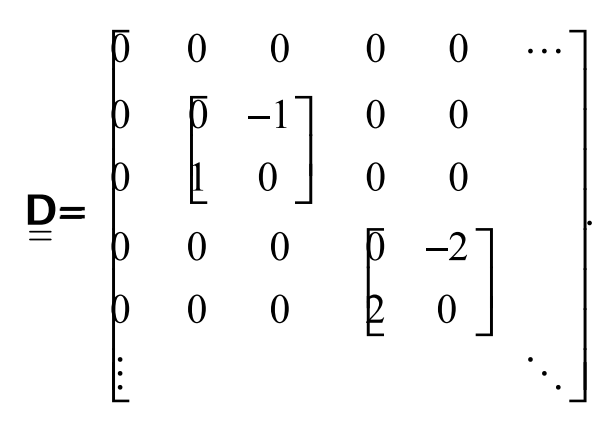

Using these operator matrices, the relationship between state variables in time domain, $\underline{z}_{j}$, and in frequency domain, $\underline{a}_{j}$, is defined,

$$
\underline{\underline{\underline{z}}}_{j}=\underline{\mathbf{F}}^{-1} \underline{a}_{j}
$$

where $\underline{a}$ is the vector of Fourier coefficients. The derivative of the state vector is also related, 


$$
\underline{\underline{\hat{z}}}_{j}^{\prime}=\underline{\mathbf{F}}^{-1} \underline{\underline{\mathbf{D}}} \underline{\underline{a}}_{j} .
$$

These equations allow the transformation of the residual into Fourier domain, $\underline{\Gamma}_{j}=\underline{\mathbf{F}} \underline{\hat{\gamma}}_{j}$, where the optimization algorithm may now be applied. Further, Newton's method is selected as the algorithm to minimize the residual. This requires the calculation of the Jacobian $(\mathrm{J})$ of the residual in Fourier domain,

$$
\underline{\mathbf{J}}_{=j, k}=\frac{\partial \underline{\Gamma}_{j}}{\partial \underline{a}_{k}}=\underset{\mathbf{F}}{=}\left[\frac{\partial \underline{\hat{\gamma}}_{j}}{\partial \underline{\hat{z}}_{k}} \frac{\partial \underline{\hat{z}}_{k}}{\partial \underline{a}_{k}}+\frac{\partial \underline{\hat{\gamma}}_{j}}{\partial \underline{\hat{z}}_{k}^{\prime}} \frac{\partial \underline{\hat{z}}_{k}^{\prime}}{\partial \underline{a}_{k}}\right]
$$

Differentiating equations (25) and (26) and substituting into equation (27) yields,

$$
{\underset{J}{j, k}}_{j, k}=\underline{\mathbf{F}}\left[\frac{\partial \underline{\hat{\gamma}}_{j}}{\partial \underline{\hat{z}}_{k}} \mathbf{F}^{-1}+\frac{\partial \underline{\hat{\gamma}}_{j}}{\partial \underline{\hat{z}}_{k}^{\prime}}=^{-1} \underline{\underline{\mathbf{D}}}\right]
$$

leaving the partial derivatives of the residual to be calculated in terms of submatrices,

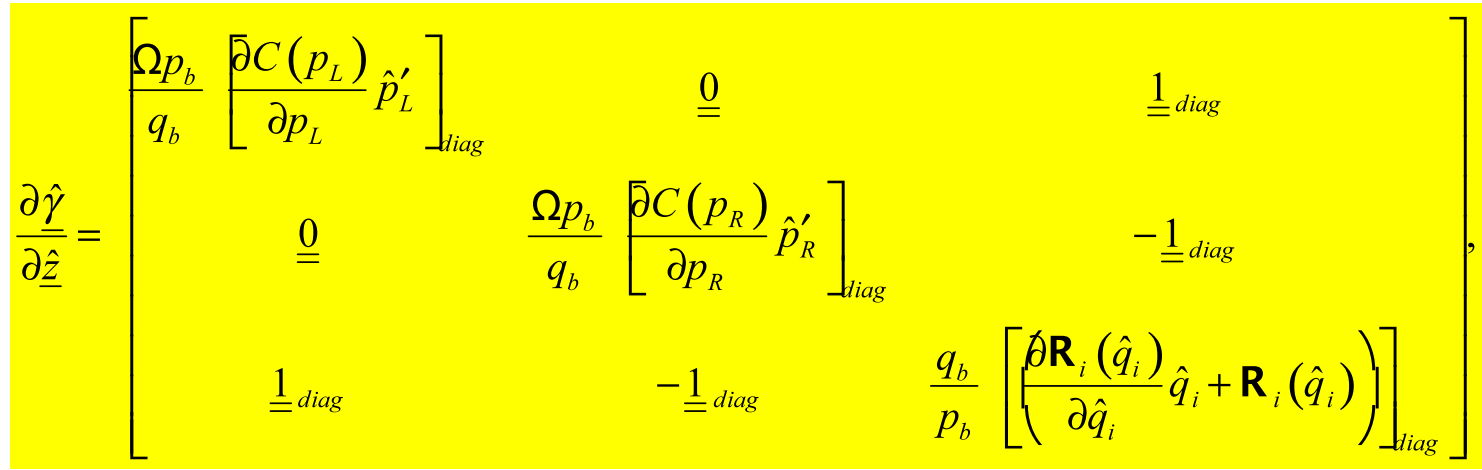




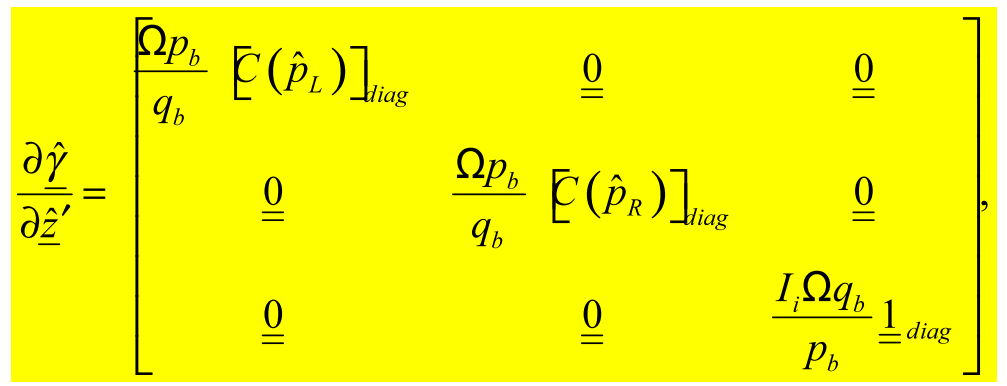

where the diag subscript indicates a diagonal matrix, in this case of dimension $M$, where $M$ is the number of points in one period of $\underline{\theta}$ and where the state variables are evaluated at each sampled point in normalized time.

An initial guess of Fourier coefficients for each state variable is needed to run the algorithm. Iteratively choosing small, normally distributed random numbers for each yields different results each time. Since pressures inside the pumping chambers of production bushings cannot be easily measured, Figure 10 shows a representative comparison between the semianalytical approximation (using a single harmonic term) with the numerical solution of the nonlinear model. Significant error exists in the mean component of each pressure term, which can be traced to a singular Jacobian matrix which has a complex-valued eigenvalue matrix $\tilde{\Lambda}_{\text {diag }}$, with distinct complex-conjugate pairs of eigenvalues, $\tilde{\lambda}_{j}$,

$$
\tilde{\Lambda}_{\text {diag }}=\operatorname{diag}\left[\begin{array}{lllllll}
\tilde{\lambda}_{1} & \tilde{\lambda}_{1}^{*} & \tilde{\lambda}_{2} & \tilde{\lambda}_{2}^{*} & 0 & \tilde{\lambda}_{3} & \tilde{\lambda}_{3}^{*}
\end{array}\right] \text {. }
$$

The zero eigenvalue acts as a blind spot in the algorithm. The mean components of both $p_{L}$ and $p_{R}$ have significant modal participation in the corresponding eigenvector, suggesting that the optimization is not sensitive to these terms. The dependence of the nonlinear compliance on chamber pressure indicates that the system is sensitive to mean pressure. An initial mean 
chamber pressure must be supplied in the numerical simulation; likewise, the initial guess of mean pressure is an external constraint rather than part of the dynamic system response.

Alternative guesses of the initial condition or average pressure from simulation yield improved results, as illustrated in Figure 11.

Substantial inaccuracy is still present in the MHBM approximation since insufficient harmonics are included to match the non-sinusoidal pressure trace. An increase in the number of harmonics improves the accuracy, as shown in Figure 12. Three harmonics produce excellent agreement with the numerically integrated pressures.

The presence of super-harmonics illustrates some of the limitations of conventional dynamic stiffness characterization methods widely used for hydraulic bushings. In sinusoidal testing procedures [24], harmonic distortion (caused by super or subharmonics) is generally eliminated by frequency-domain filtering or sine regression techniques, reducing the dynamic behavior to a seemingly linear response at a given amplitude. Analyzing the superharmonic content of measured transmitted force signals (if available) may improve experimental insight, particularly when compared to the signatures produce by reduced order models and the MHBM.

Finally, note that the MHBM is able to produce a solution approximation, yielding frequency responses and harmonic decomposition even in unstable and multi-valued regimes. This requires much less computational time when compared to "brute force" numerical methods. While unstable and multi-valued regimes do not seem to be present in this study, another device with different parameters may demonstrate such behavior. Therefore, the semi-analytical technique adds robustness to the proposed methods as well as providing additional insight.

\section{Comparative evaluation of 4 models}


Four hydraulic bushing models are considered to address the amplitude sensitivity of $K(\Omega)$ in both magnitude and loss angle spectra: quasi-linear model (I) and nonlinear models, specifically models containing only a nonlinear resistance parameter (II), only a nonlinear compliance parameter (III), and a model containing both nonlinear resistance and compliance elements (IV). The error in each model $\varepsilon_{K}$ is quantified, where $M_{\Omega}$ is the number of frequencies considered,

$$
\varepsilon_{K_{I I}}=\frac{1}{K_{r} M_{\Omega}} \sqrt{\sum_{j=1}^{M_{\Omega}}\left\|\tilde{K}_{I I}\left(\Omega_{j}\right)-\tilde{K}_{d}\left({ }_{j}\right)\right\|^{2}},
$$

taking model II as an example. The error is normalized by the static (rubber path) and the accuracy of each model is compared in terms of this error in Table 2.

The quasi-linear bushing model produces reasonable results capturing amplitude sensitivity, but they lack the ability to predict the trends in amplitude sensitivity. Nonlinear models remove the need for dynamic stiffness measurements at many amplitudes. The nonlinear resistance model (II) presents significantly high error, heavily weighted towards the lower excitation amplitudes. Model III (with nonlinear compliance) yields somewhat reduced error, particularly at lower amplitudes because the linear resistance parameter was taken from Model I with $x_{a}=0.1 \mathrm{~mm}$. The interaction between the two nonlinear elements in model IV generally reduces the error across all amplitudes, suggesting that the inclusion of nonlinear compliance is necessary but not sufficient to capture the physics of this device. Further extension to characterize a nonlinear effective pumping area parameter, $A(x, p)$, would be a logical step since the excitation to the fluid system is proportional to $A$. 
Use of the multi-term harmonic balance method offers some insight into the physics of the system, since each of the harmonic terms may be examined. The method produces coefficients for sine and cosine terms at each harmonic, which are easily converted to sinusoidal magnitudes and phases. Figure 13 shows the amplitudes of the first harmonic pressure term at each excitation amplitude. The shape of these pressure spectra in some ways resembles the dynamic stiffness plots in Figure 7 because the fluid force path contribution becomes most significant especially when the fluid system nears its effective resonance. Although this is a nonlinear system, the corresponding linearized fluid system has a single-valued resonance, so the response amplitudes increase significantly near that frequency.

The amplitude sensitivity of superharmonic pressure magnitudes in model IV is depicted in Figure 14, while the phases are compared in Figure 15. Two peaks are visible in the magnitude of the third harmonic at $0.1 \mathrm{~mm}$, but the second peak begins to flatten with increasing amplitude while the first peak seems unaffected. The second peak occurs at $\hat{\Omega}_{f}=1.25$, which corresponds to the peak in the first harmonic and represents the resonant frequency of the linearized fluid system. The first peak occurs at $\hat{\Omega}=0.44$, which is the closest point to $\hat{\Omega}_{f} / 3$. To sort out which nonlinearity is causing these effects, the harmonic terms in models II and III are compared.

The first three pressure harmonics for models II, III, and IV at $0.1 \mathrm{~mm}$ excitation amplitude are compared in Figure 16. The magnitude of the second pressure harmonic in Model II is several orders of magnitude smaller than the first and third harmonics, so effectively, only a third harmonic exists. At first glance, it appears that the superharmonic terms in model IV are simply a superposition of the superharmonics from models II and III, though the superposition 
principle should not apply to nonlinear systems. Instead, the observed effect represents an interaction between the $R_{i}\left(q_{i}\right)$ and $C(p)$ nonlinearities in the system. This result highlights the insufficiency of Model II, which only produces a third harmonic. This is most likely because the exponent on the state variable $q_{i}$ is 1.75 , which is very close to $\sqrt{3} \approx 1.73$, producing a third

harmonic in the response. Also, neither model II nor III captures the regime change near $\hat{\Omega}=0.6$ where a shift in the dominant harmonic suggests that a different physical effect controls the system response. Below this frequency, the third harmonic is much higher than the second, indicating that $R_{i}\left(q_{i}\right)$ is the dominant nonlinearity. Beyond this frequency, the second harmonic is larger, suggesting that $C(p)$ becomes the dominant effect, somewhat analogous to the mass or stiffness controlled regimes of a simple mechanical oscillator. The absence of a peak in the second harmonic near $\hat{\Omega}=0.44$ is explained because $C(p)$ contains linear, quadratic, and cubic terms, which prevents significant resonant excitation at exactly one half or one third of the fluid system's linearized resonant frequency.

\section{Conclusion}

This article compares two modeling approaches to predict and explain the amplitude dependent dynamic stiffness spectra of a hydraulic bushing. Unlike the prior work [17] on bushing models that focused on a laboratory device and used only a nonlinear resistance parameter, this article describes a production bushing, which makes the parameter estimation a more difficult process. A quasi-linear model is first proposed which is based on a curve-fit of measured dynamic stiffness using an assumed transfer function form of the governing system. The effect of amplitude on each physical parameter is observed, but the trends offer minimal 
physical insight. Since dynamic stiffness measurements are inherently required at each amplitude of interest, the utility of the quasi-linear approach is limited, especially when a new device is to be designed. Consequently, alternate nonlinear models must be considered and implemented for up-front design and diagnostics work to meet increasingly stringent vehicle requirements. Nonlinear models better capture the trends in amplitude sensitivity, provide physical insight, and eliminate the need for dynamic testing at all amplitudes since the model's amplitude sensitivity is not based on empirical stiffness data. Nonlinear compliance elements are used, which interacts with the nonlinear resistance element to improve the amplitude sensitivity predictions. The MHBM procedure of Chai et al. [17] is also refined with improved generating solution approximations for the new system equations which contain both nonlinear compliance and resistance elements.

In summary, this article extends the literature [15-18], and the specific contributions include (1) new and experimentally validated quasi-linear and nonlinear models which improve amplitude sensitivity in dynamic stiffness predictions, and (2) a refined semi-analytical method (MHBM) which captures and offers physical insight into multiple interacting nonlinearities in a system of three equations. The results suggest that nonlinear models of resistance and compliance are necessary. While these two nonlinear elements yield reasonable dynamic stiffness predictions, further work developing a nonlinear pumping area expression (as given by equation (13)) should improve characterization of dynamic stiffness amplitude sensitivity.

\section{Acknowledgement}

We acknowledge the member organizations of the Smart Vehicles Concepts Center (www.SmartVehicleCenter.org) such as Transportation Research Center Inc., Honda R\&D 
Americas, Inc., F.tech R\&D North America, Inc., Tenneco, Inc. and the National Science

Foundation Industry/University Cooperative Research Centers program

(www.nsf.gov/eng/iip/iucrc) for supporting this work.

\section{References}

[1] J.Y. Yoon and R. Singh, Indirect measurement of dynamic force transmitted by a nonlinear hydraulic mount under sinusoidal excitation with focus on super-harmonics, J. Sound Vib. 329 (2010) 5249-5272.

[2] J.-Y. Yoon and R. Singh, Estimation of interfacial forces in a multi-degree of freedom isolation system using a dynamic load sensing mount and quasi-linear models, J. Sound Vib. 330 (2011) 4429-4446.

[3] S. He and R. Singh, Estimation of amplitude and frequency dependent parameters of hydraulic engine mount given limited dynamic stiffness measurements, Noise Control Eng. J. 53(6) (2006) 255-269.

[4] M. Tiwari, H. Adiguna, and R. Singh, Experimental characterization of a nonlinear hydraulic engine mount, Noise Control Eng. J. 51(1) (2003) 36-49.

[5] G. Kim and R. Singh, Nonlinear analysis of automotive hydraulic engine mount, ASME J. Dyn. Syst. Meas. Control 115 (1993) 482-487.

[6] J. H. Lee and R. Singh, Non-linear frequency responses of quarter vehicle models with amplitude-sensitive engine mounts, J. Sound Vib. 313 (2008) 784-805.

[7] W. B. Shangguan and Z. H. Lu, Modelling of a hydraulic engine mount with fluid—structure interaction finite element analysis, J. Sound Vib. 275(1) (2004) 193-221.

[8] J. Christopherson and R. N. Jazar, Dynamic behavior comparison of passive hydraulic engine mounts. Part 1: mathematical analysis, J. Sound Vib. 290(3) (2006) 1040-1070. 
[9] J. Christopherson and R. N. Jazar, Dynamic behavior comparison of passive hydraulic engine mounts. Part 2: finite element analysis, J. Sound Vib. 290(3) (2006) 1071-1090.

[10] W. Sauer, Y. Guy, Hydro bushings - innovative NVH solutions in chassis technology. SAE Paper 2003-01-1475 (2003).

[11] N. Gil-Negrete, A. Rivas, and J. Vinolas, Predicting the dynamic behaviour of hydro bushings, Shock Vib. 12 (2005) 91-107.

[12] S. Arzanpour, M. Golnaraghi, Development of a bushing with an active compliance chamber for variable displacement engines, Vehicle Syst. Dyn. 46 (2008) 867-887.

[13] T. Chai, J.T. Dreyer, and R. Singh, Transient response of hydraulic bushing with inertia track and orifice-like elements, SAE Int. J. Passeng. Cars - Mech. Syst. 6 (2013) 1224-1230. [14] T. Chai, R. Singh, and J. T. Dreyer, Dynamic stiffness of hydraulic bushing with multiple internal configurations, SAE Int. J. Passeng. Cars - Mech. Syst. 6 (2013) 1209-1216.

[15] T. Chai, J.T. Dreyer, and R. Singh, Frequency domain properties of hydraulic bushing with long and short passages: system identification using theory and experiment, Mech. Syst. Signal Process 56 (2015) 92-108.

[16] T. Chai, J.T. Dreyer, and R. Singh, Time domain responses of hydraulic bushing with two flow passages, J. Sound Vib. 333 (2014) 693-710.

[17] T. Chai, J.T. Dreyer, and R. Singh. Nonlinear dynamic properties of hydraulic suspension bushing with emphasis on the flow passage characteristics, Proc. IMechE Part D: J. Automob. Eng. (2014): 0954407014561048.

[18] L. Fredette, J.T. Dreyer, and R. Singh, Dynamic analysis of hydraulic bushings with measured nonlinear compliance parameters, SAE Int. J. Passeng. Cars - Mech. Syst. 8 (2015) 2015-01-2355 
[19] T.C. Kim, T.E. Rook and R. Singh, Super- and sub-harmonic response calculations for a torsional system with clearance non-linearity using harmonic balance method, J. Sound Vib. $281(3)(2005)$ 965-993.

[20] S. He, T. Rook and R. Singh, Construction of semianalytical solutions to spur gear dynamics given periodic mesh stiffness and sliding friction functions, ASME J. of Mech.Des. 130 (12) (2008) 122601.

[21] MATLAB Release 2013b, The MathWorks, Inc., Natick, Massachusetts, United States.

[22] E. Wyle, V. Streeter, Fluid transients, McGraw-Hill, 1978.

[23] E. O. Doebelin, System dynamics: modeling, analysis, simulation, design, New York: Marcel Dekker, 1998.

[24] MTS Systems Corporation, Model 793.31 Dynamic Characterization Process - Operating Manual, 2000.

\section{Figure Captions:}

Figure 1. Hydraulic bushing model. $F_{T}$ is the transmitted force, $x(t)$ is the displacement excitation, $K_{r}$ is the stiffness and $\mathrm{c}_{r}$ is the damping of the rubber path. For the pumping chambers, $C$ is the fluid compliance, $A$ is the effective pumping area, and $p$ is the absolute pressure in each chamber, right $(R)$ and left $(L)$. In the inertia track, $I_{i}$ is the fluid inertance, $\mathbf{R}_{i}$ is the fluid resistance and $q_{i}$ is the volume flow rate. 
Figure 2. Measured dynamic stiffness magnitude (top) and loss angle (bottom) of a production bushing showing significant sensitivity to excitation amplitude. Key: $-x_{a}=0.1 \mathrm{~mm} ;---x_{a}=$ $0.5 \mathrm{~mm} ;-\cdot x_{a}=1.0 \mathrm{~mm} ; \cdots x_{a}=2.0 \mathrm{~mm}$ peak to peak excitation amplitude.

Figure 3. Comparison of quasi-linear models and measured dynamic stiffness magnitudes at various peak to peak excitations: $(a) x_{a}=0.1 \mathrm{~mm},(b) x_{a}=0.5 \mathrm{~mm},(c) x_{a}=1.0 \mathrm{~mm},(d) x_{a}=2.0$ mm. Key: - Measured; --- transfer function coefficient curve-fit; '.... physical parameter curve-fit.

Figure 4. Comparison of quasi-linear models and measured loss angles of the production bushing at various peak to peak excitations: $(a) x_{a}=0.1 \mathrm{~mm},(b) x_{a}=0.5 \mathrm{~mm},(c) x_{a}=1.0 \mathrm{~mm}$, (d) $x_{a}=2.0 \mathrm{~mm}$. Key: - Measured; --- transfer function coefficient curve-fit; '-.... physical parameter curve-fit.

Figure 5. Measured pressure dependent volume of the pumping chambers on a production bushing, and least squares cubic curve fit. Key: * Measured data from bench experiment; least squares curve fit.

Figure 6. Nonlinear compliance $C(p)$ compared with linear compliance $C$ identified from empirical quasi-linear modeling with a $0.1 \mathrm{~mm}$ amplitude. Parameters are normalized by the linear compliance. Key: - Nonlinear $C(p)$; --- Linear $C$. 
Figure 7. Predicted stiffness spectra of Model IV. Key: $-x_{a}=0.1 \mathrm{~mm} ;---x_{a}=0.5 \mathrm{~mm} ;-\cdot-$ $x_{a}=1.0 \mathrm{~mm} ; \cdots x_{a}=2.0 \mathrm{~mm}$.

Figure 8. Comparison between amplitude dependent measured and predicted dynamic stiffness magnitudes: $(a) x_{a}=0.1 \mathrm{~mm},(b) x_{a}=0.5 \mathrm{~mm},(c) x_{a}=1.0 \mathrm{~mm},(d) x_{a}=2.0 \mathrm{~mm}$. Key: --- $\left|\hat{K}_{I I}\right| ; \cdot \cdot-\cdot\left|\hat{K}_{I I I}\right| ; \cdots \cdots\left|\hat{K}_{I V}\right| ;-\left|\hat{K}_{d}\right| \cdot$

Figure 9. Comparison between measured and predicted loss angles at various excitation amplitudes: $(a) x_{a}=0.1 \mathrm{~mm},(b) x_{a}=0.5 \mathrm{~mm},(c) x_{a}=1.0 \mathrm{~mm},(d) x_{a}=2.0 \mathrm{~mm}$. Key: --- $\phi_{I I} ; \cdot-\cdot \phi_{I I} ; \cdots \cdots \phi_{I V} ;-\phi_{d}$.

Figure 10. Representative comparison between nonlinear simulation results and solution approximation generated with single-term harmonic balance method (HBM) and normally distributed random values for the initial guess of Fourier coefficients. Very poor accuracy is achieved. Key: - Simulated $p_{L} ;$ Simulated $p_{R} ;---\operatorname{HBM} p_{L} ;$--- HBM $p_{R}$.

Figure 11. Representative comparison between nonlinear simulation results and solution approximation generated with the single-term harmonic balance method (HBM). (a) Initial guess for mean pressure taken from initial condition in numerical simulation. (b) Initial guess for mean pressure taken by averaging simulated pressure traces. Marginal accuracy is achieved. Key: Simulated $p_{L} ;-$ Simulated $p_{R} ;---\operatorname{HBM} p_{L} ;$--- HBM $p_{R}$. 
Figure 12. Representative comparison between simulation results and solution approximation generated with the multi-term harmonic balance method using multiple harmonics. (a) Two harmonics. Improved accuracy is achieved. (b) Three harmonics. Excellent accuracy is achieved. Key: - Simulated $p_{L} ;-$ Simulated $p_{R} ;---\operatorname{MHBM} p_{L} ;---\operatorname{MHBM} p_{R}$.

Figure 13. Amplitude of the fundamental (first) pressure harmonic of Model IV. Key: - $x_{a}=$ $0.1 \mathrm{~mm} ;---x_{a}=0.5 \mathrm{~mm} ;-\cdot-x_{a}=1.0 \mathrm{~mm} ; \cdots x_{a}=2.0 \mathrm{~mm}$.

Figure 14. Existence and relative magnitudes of super harmonics as illustrated by harmonic pressure terms in the nonlinear dynamic responses. Sine and cosine coefficients are calculated by the multi-term harmonic balance method, converted to magnitude form, and normalized by excitation amplitude. (a) $x_{a}=0.1 \mathrm{~mm},(b) x_{a}=0.5 \mathrm{~mm},(c) x_{a}=1.0 \mathrm{~mm}$, and (d) $x_{a}=2.0 \mathrm{~mm}$. Key: — First harmonic; --- second harmonic; ...... third harmonic.

Figure 15. Existence and relative phases of super harmonics as illustrated by harmonic pressure terms in the nonlinear dynamic responses. Sine and cosine coefficients are calculated by the multi-term harmonic balance method and converted to phase form at several excitation amplitude, (a) $x_{a}=0.1 \mathrm{~mm},(b) x_{a}=0.5 \mathrm{~mm},(c) x_{a}=1.0 \mathrm{~mm}$, and $(d) x_{a}=2.0 \mathrm{~mm}$. Key: First harmonic; --- second harmonic; ...... third harmonic. 
Figure 16. Relative magnitudes of super harmonics as illustrated by harmonic pressure terms in the nonlinear dynamic responses. Sine and cosine coefficients are calculated by the multi-term harmonic balance method, converted to magnitude form, and normalized by excitation

amplitude. (a) Model II, (b) Model II, and (c) Model IV. Key: — First harmonic; --- second harmonic; $\cdots . .$. third harmonic.

\section{Table Captions:}

Table 1. Effect of displacement excitation amplitude $\left(x_{a}\right)$ on the parameters of quasi-linear model (I). Parameters are normalized by their nominal values with a $0.1 \mathrm{~mm}$ excitation.

Table 2. Quantification of error, $\varepsilon_{K}$, associated with quasilinear (I) and nonlinear (II, III, and IV) models. 


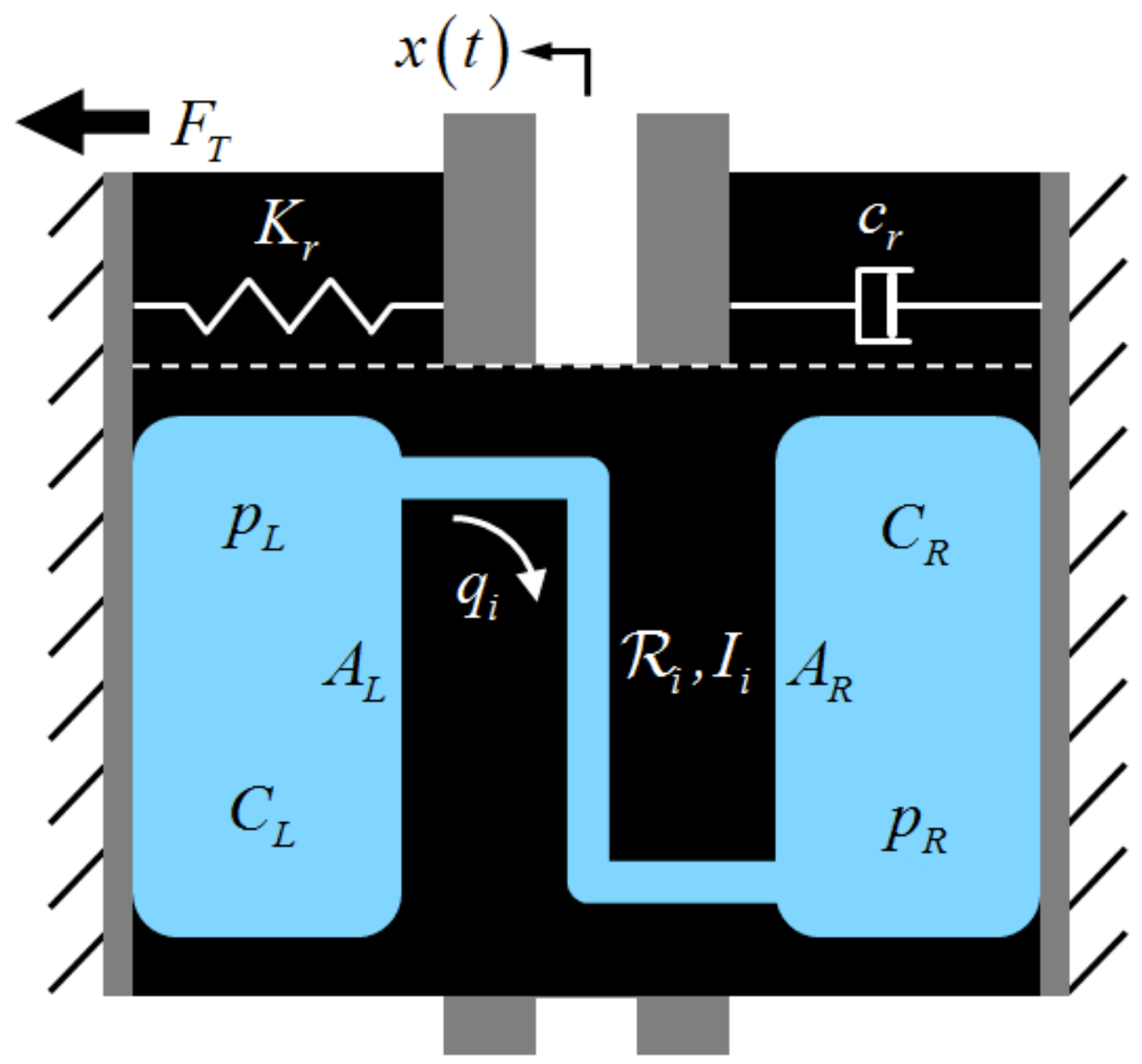

Figure 1. Hydraulic bushing model. $F_{T}$ is the transmitted force, $x(t)$ is the displacement excitation, $K_{r}$ is the stiffness and $\mathrm{c}_{r}$ is the damping of the rubber path. For the pumping chambers, $C$ is the fluid compliance, $A$ is the effective pumping area, and $p$ is the absolute pressure in each chamber, right $(R)$ and left $(L)$. In the inertia track, $I_{i}$ is the fluid inertance, $\mathbf{R}_{i}$ is the fluid resistance and $q_{i}$ is the volume flow rate. 

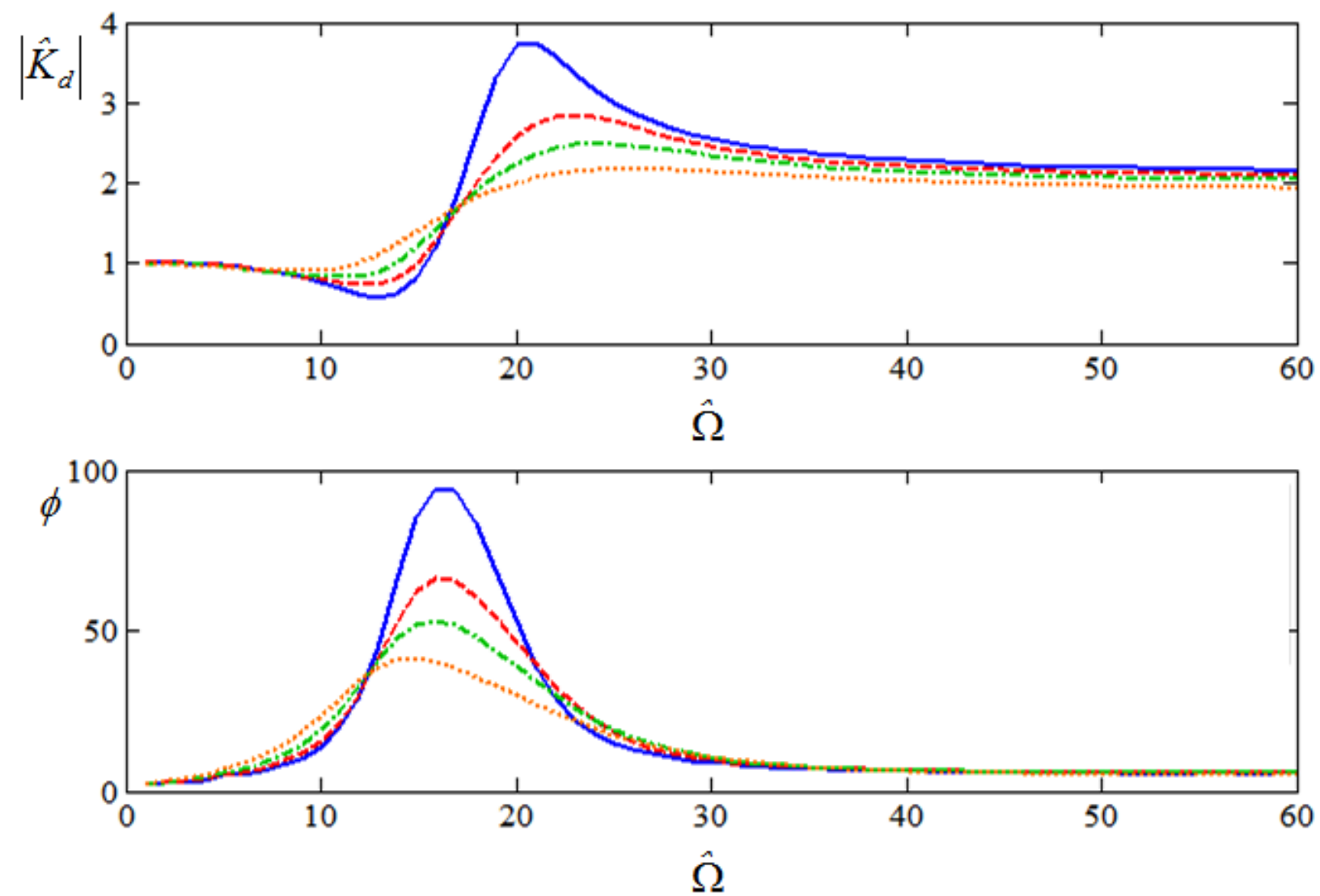

Figure 2. Measured dynamic stiffness magnitude (top) and loss angle (bottom) of a production bushing showing significant sensitivity to excitation amplitude. Key: $-x_{a}=0.1 \mathrm{~mm} ;---x_{a}=$ $0.5 \mathrm{~mm} ;{ }^{-\cdot-} x_{a}=1.0 \mathrm{~mm} ; \cdots x_{a}=2.0 \mathrm{~mm}$ peak to peak excitation amplitude. 

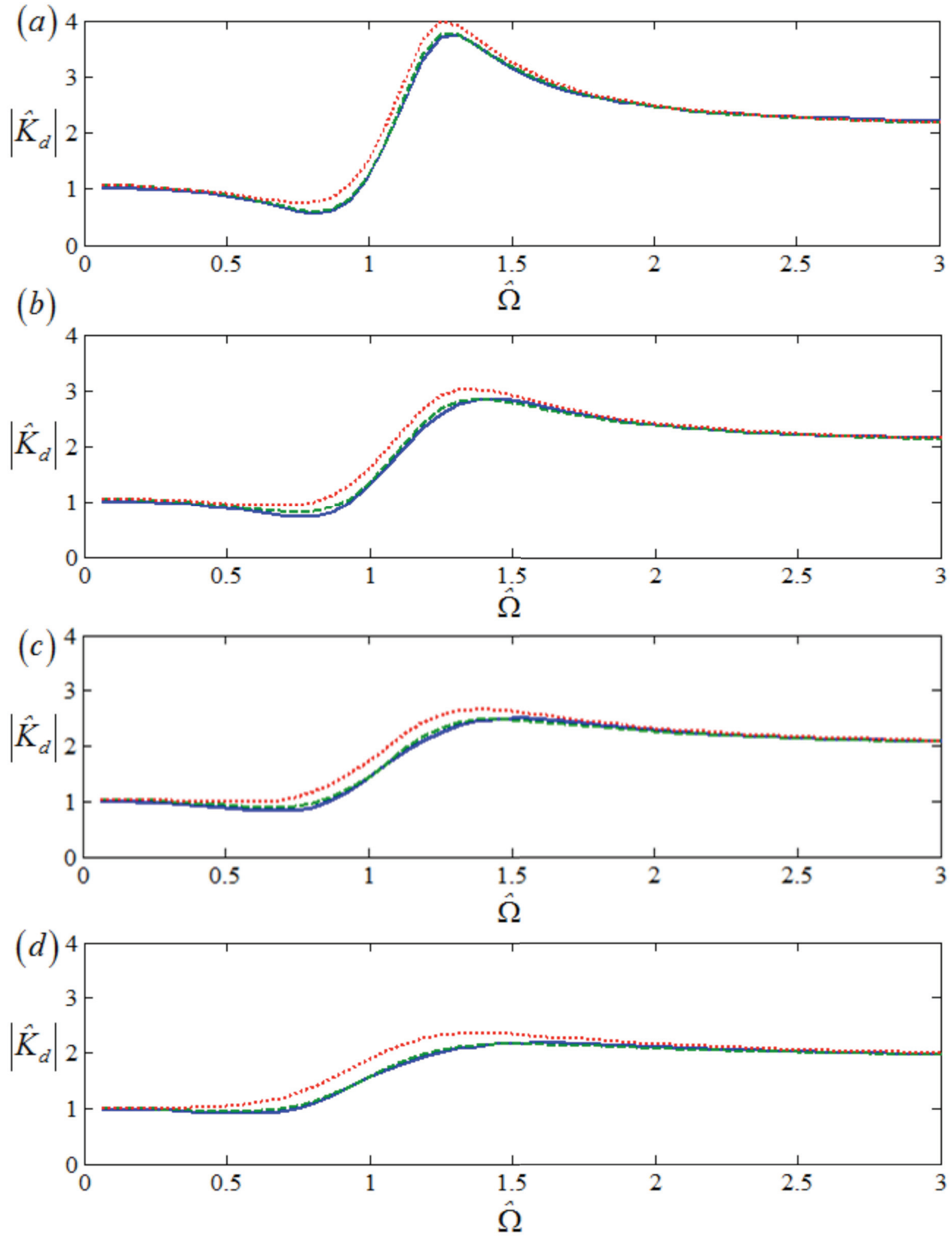

Figure 3. Comparison of quasi-linear models and measured dynamic stiffness magnitudes at various peak to peak excitations: $(a) x_{a}=0.1 \mathrm{~mm},(b) x_{a}=0.5 \mathrm{~mm},(c) x_{a}=1.0 \mathrm{~mm},(d) x_{a}=2.0$ 
mm. Key: - Measured; --- transfer function coefficient curve-fit; '… physical parameter curve-fit.
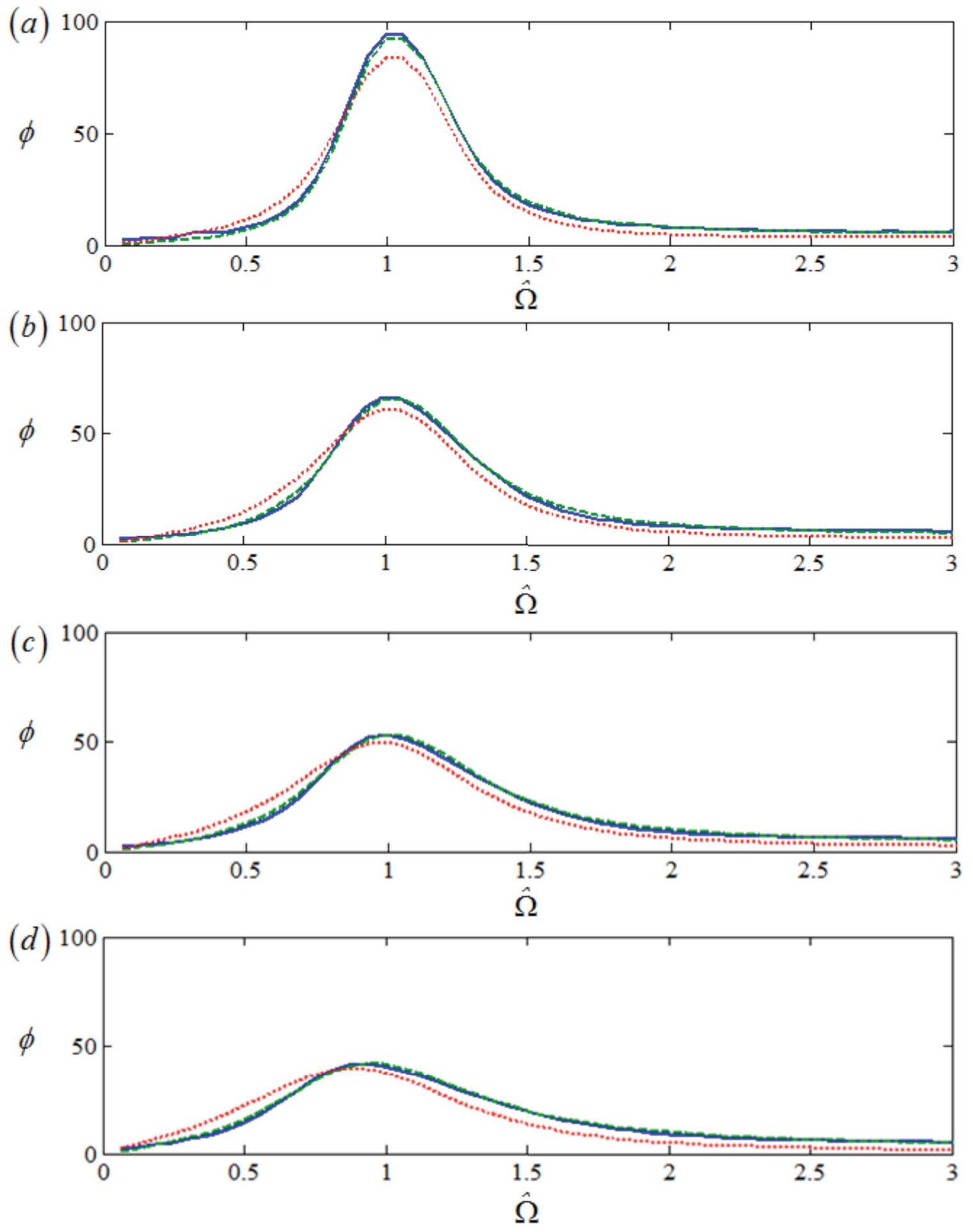
Figure 4. Comparison of quasi-linear models and measured loss angles of the production bushing at various peak to peak excitations: $(a) x_{a}=0.1 \mathrm{~mm},(b) x_{a}=0.5 \mathrm{~mm},(c) x_{a}=1.0 \mathrm{~mm}$, (d) $x_{a}=2.0 \mathrm{~mm}$. Key: - Measured; --- transfer function coefficient curve-fit; '..... physical parameter curve-fit.

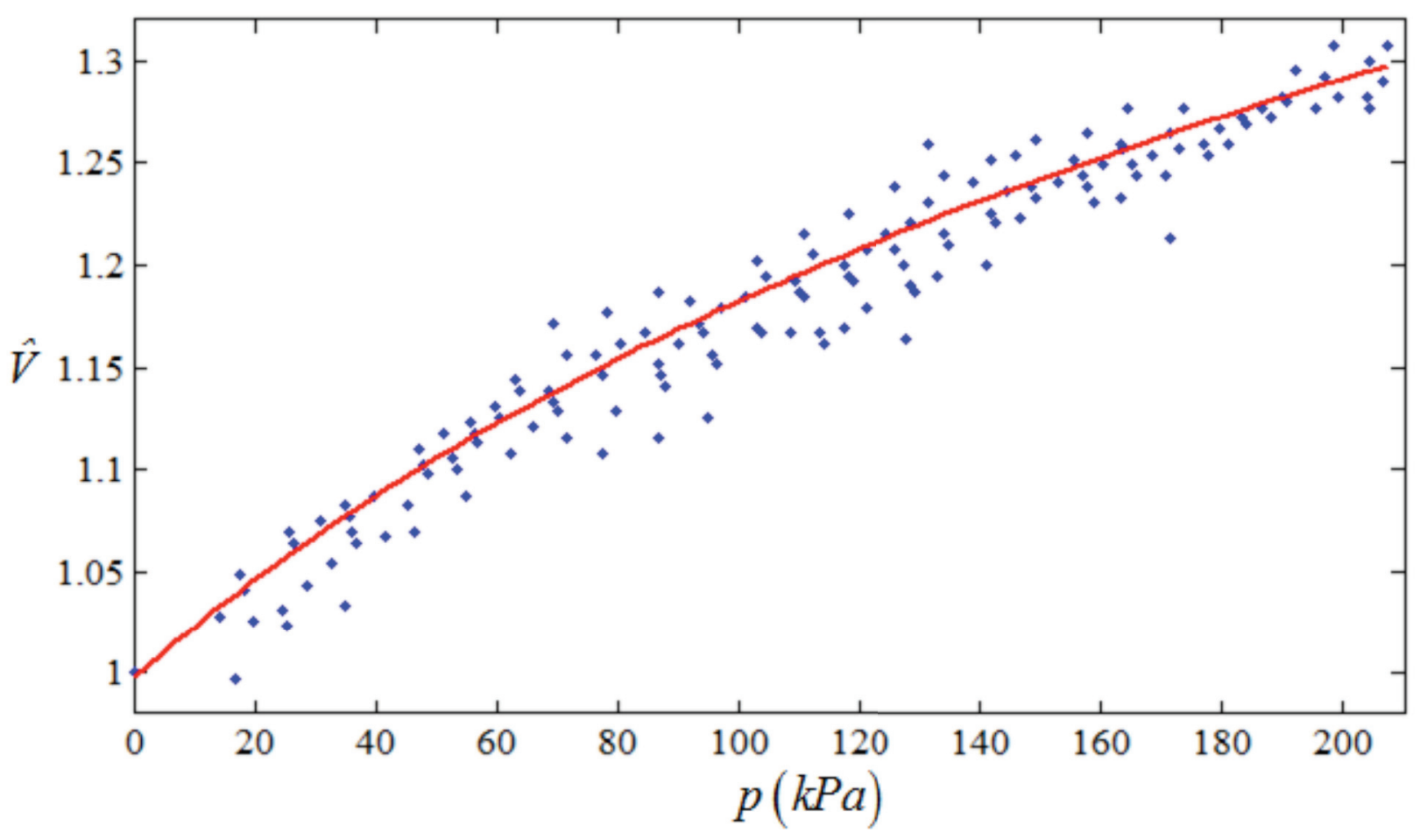

Figure 5. Measured pressure dependent volume of the pumping chambers on a production bushing, and least squares cubic curve fit. Key: * Measured data from bench experiment; least squares curve fit. 


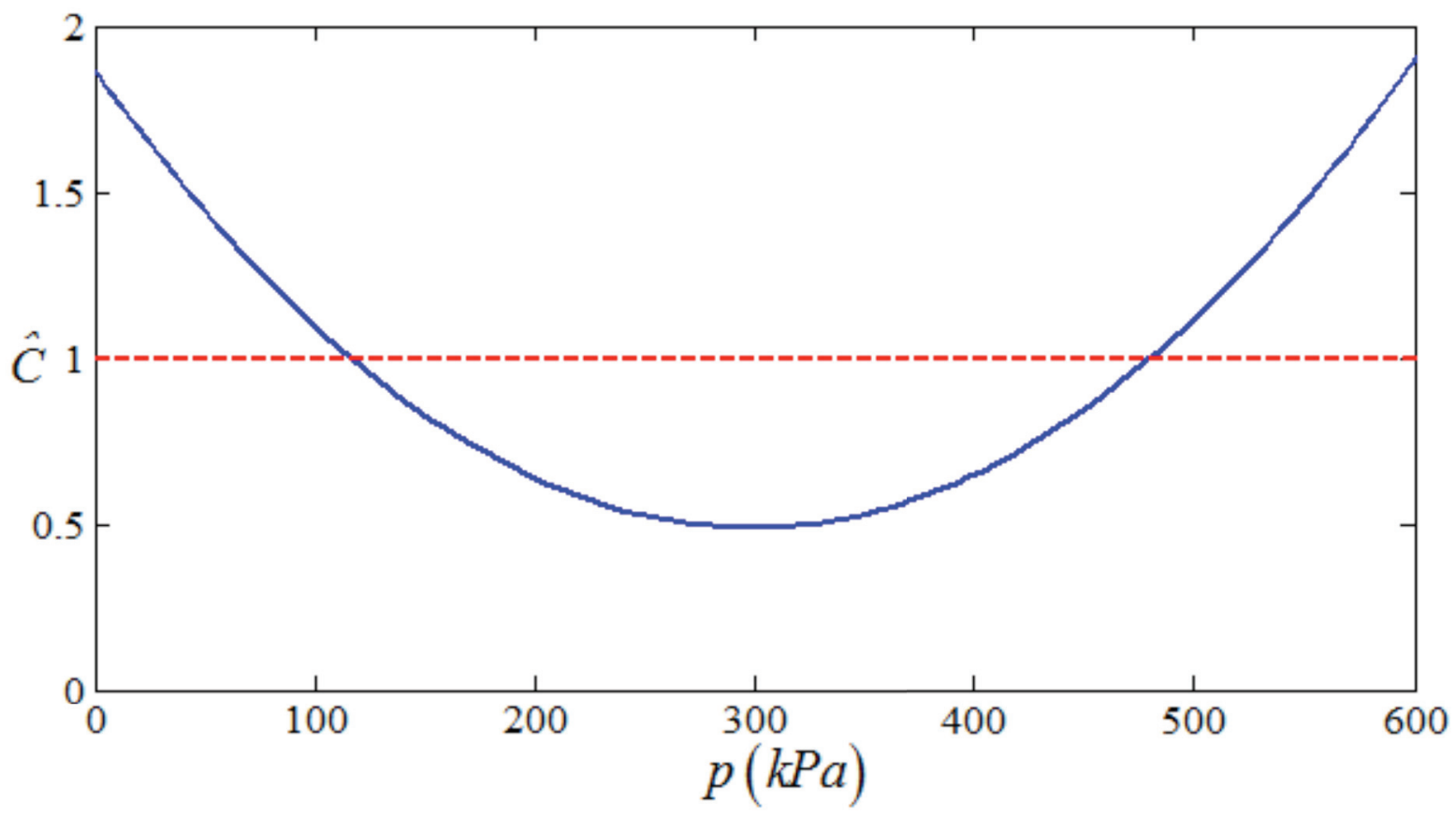

Figure 6. Nonlinear compliance $C(p)$ compared with linear compliance $C$ identified from empirical quasi-linear modeling with a $0.1 \mathrm{~mm}$ amplitude. Parameters are normalized by the linear compliance. Key: - Nonlinear $C(p)$; --- Linear $C$. 

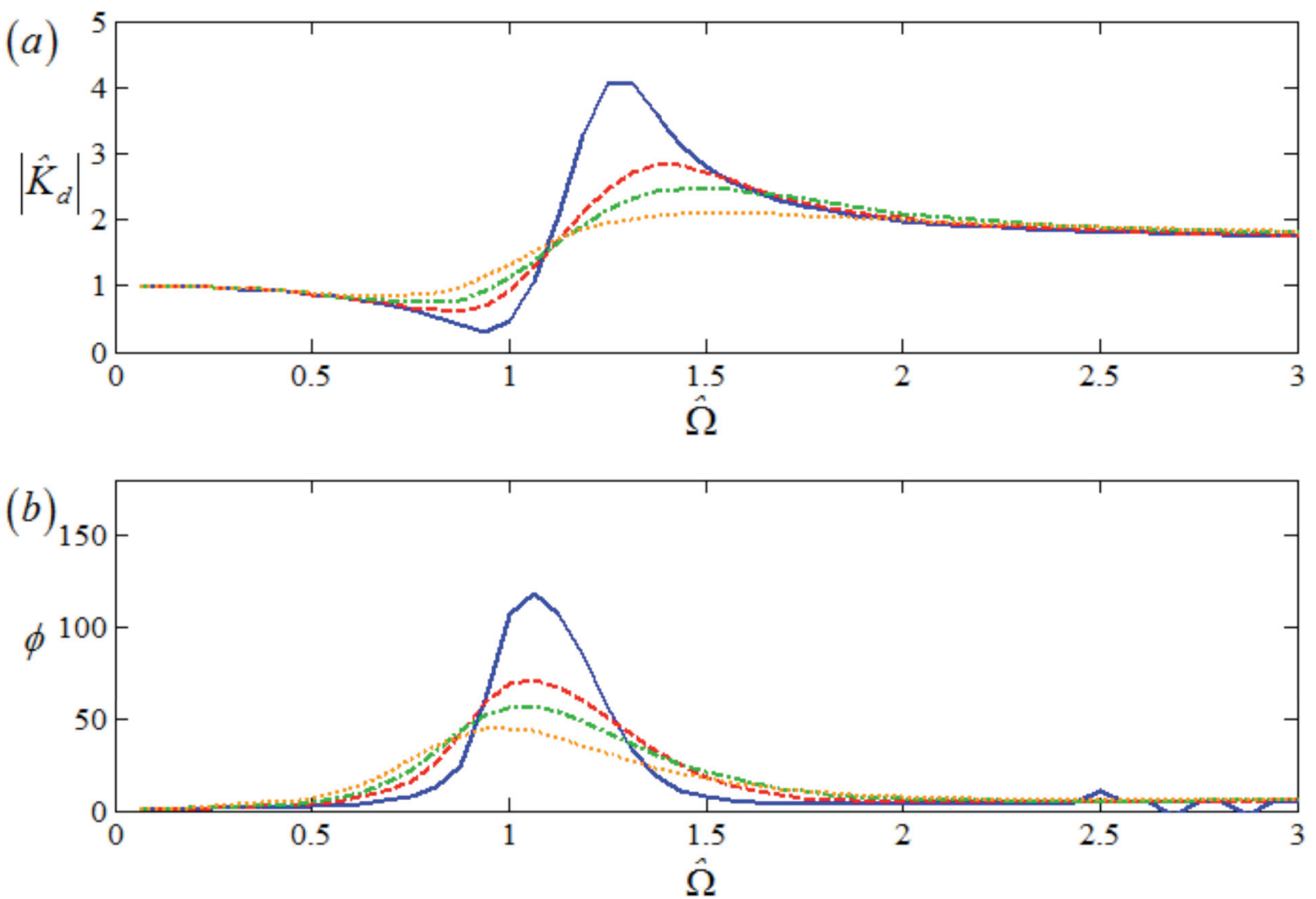

Figure 7. Predicted stiffness spectra of Model IV. Key: $-x_{a}=0.1 \mathrm{~mm} ;---x_{a}=0.5 \mathrm{~mm}$; --$x_{a}=1.0 \mathrm{~mm} ; \cdots x_{a}=2.0 \mathrm{~mm}$. 

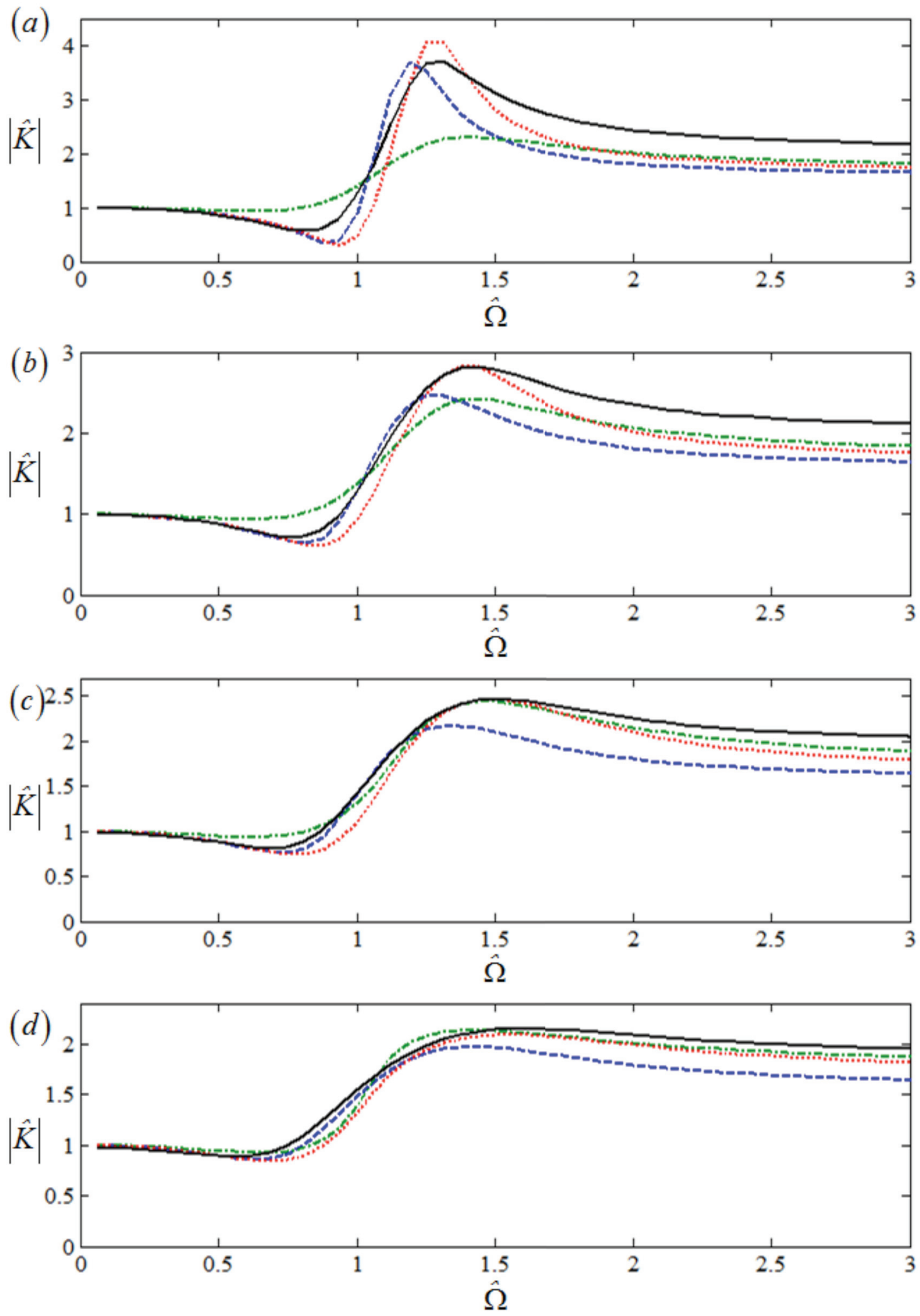

Figure 8. Comparison between amplitude dependent measured and predicted dynamic stiffness magnitudes: $(a) x_{a}=0.1 \mathrm{~mm},(b) x_{a}=0.5 \mathrm{~mm},(c) x_{a}=1.0 \mathrm{~mm},(d) x_{a}=2.0 \mathrm{~mm}$. Key: 


$$
\text { --- }\left|\hat{K}_{I I}\right| ; \cdot \cdot \cdot-\left|\hat{K}_{I I I}\right| ; \cdots \cdots\left|\hat{K}_{I V}\right| ;-\left|\hat{K}_{d}\right| \cdot
$$
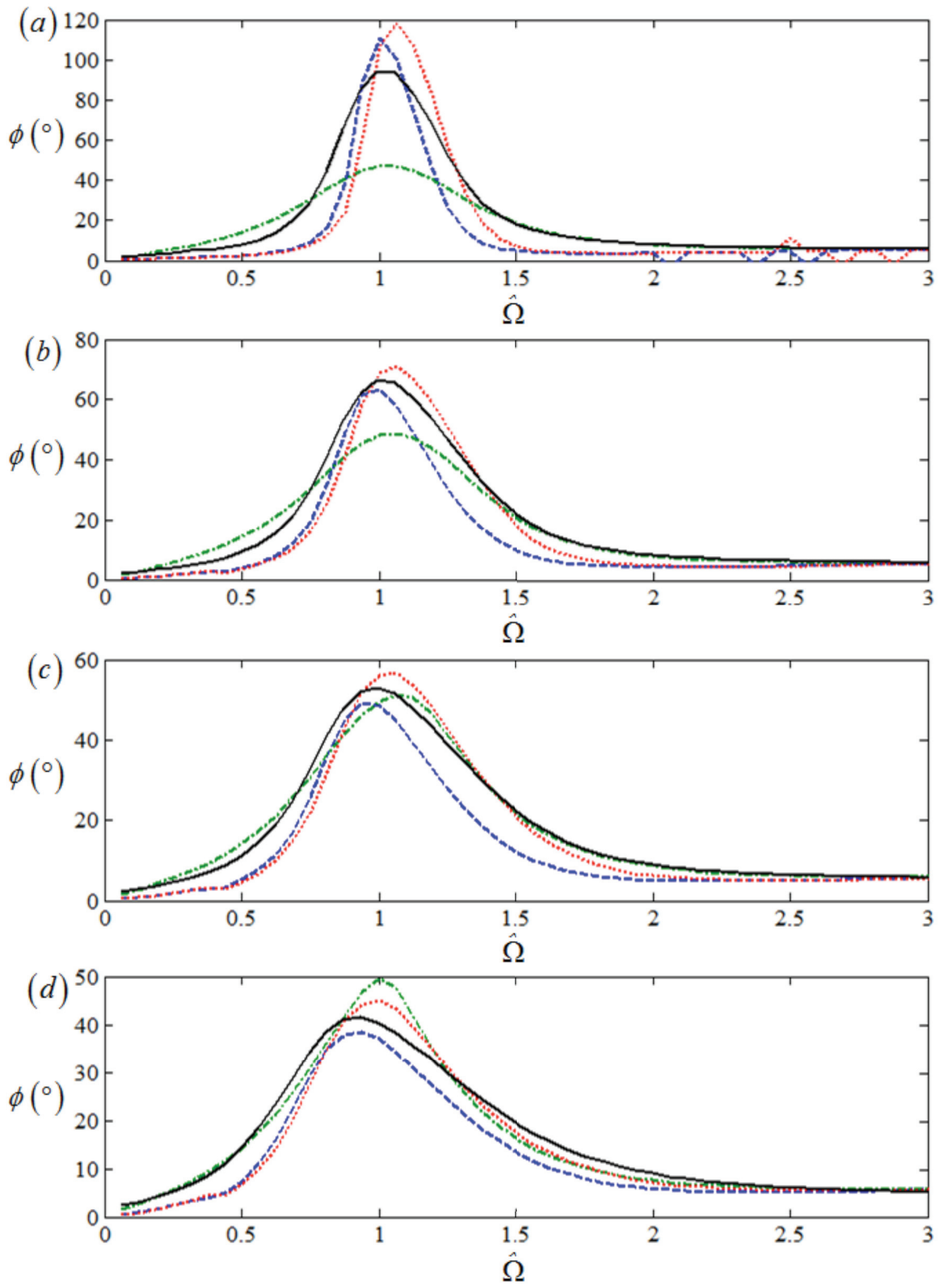
Figure 9. Comparison between measured and predicted loss angles at various excitation amplitudes: $(a) x_{a}=0.1 \mathrm{~mm},(b) x_{a}=0.5 \mathrm{~mm},(c) x_{a}=1.0 \mathrm{~mm},(d) x_{a}=2.0 \mathrm{~mm}$. Key:

$$
\text { --- } \phi_{I I} ; \cdot-\cdot \phi_{I I I} ; \cdots \cdots \phi_{I V} ;-\phi_{d} \text {. }
$$

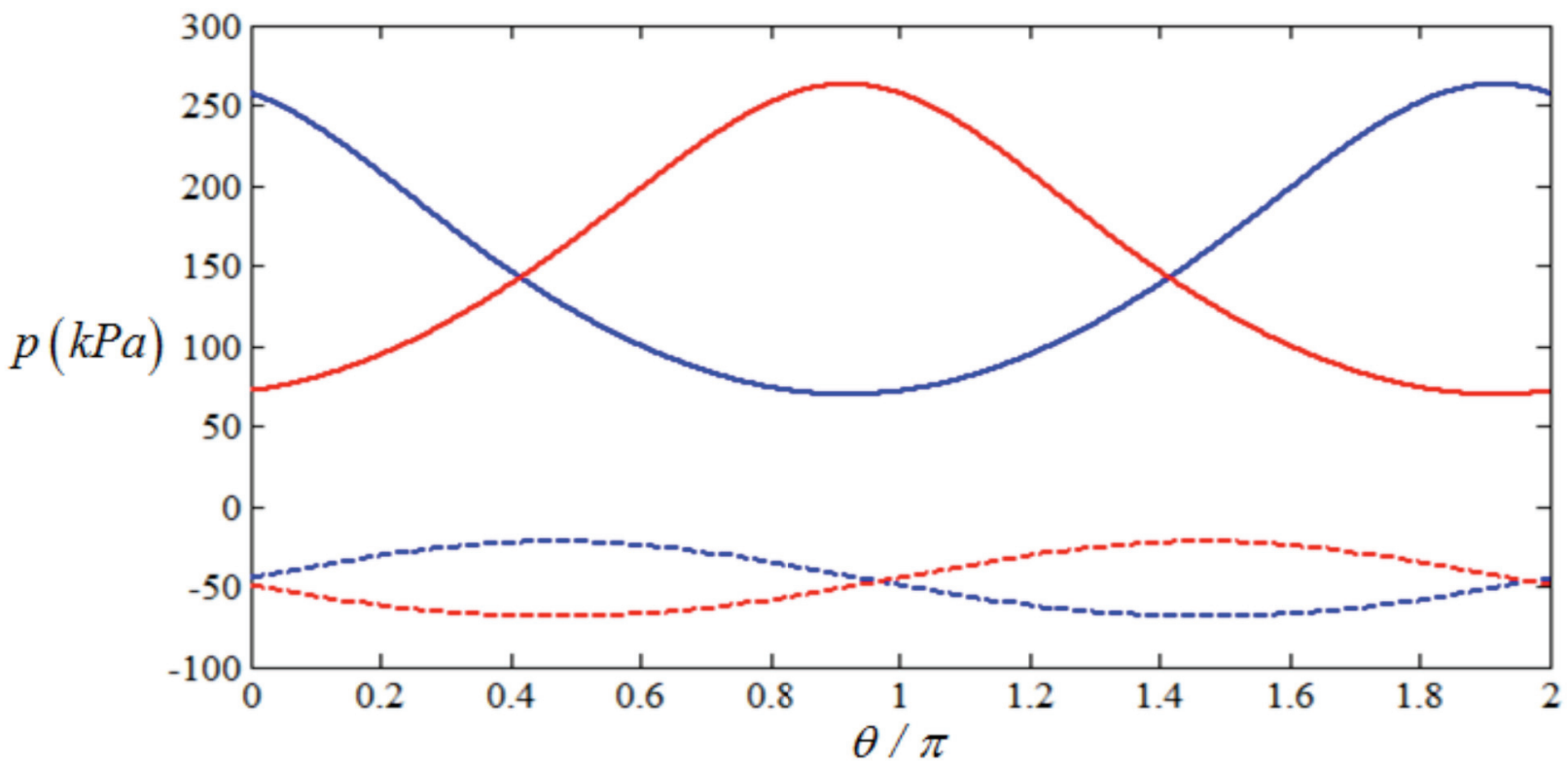

Figure 10. Representative comparison between nonlinear simulation results and solution approximation generated with single-term harmonic balance method (HBM) and normally distributed random values for the initial guess of Fourier coefficients. Very poor accuracy is achieved. Key: - Simulated $p_{L} ;-$ Simulated $p_{R} ;---\operatorname{HBM} p_{L} ;---\operatorname{HBM} p_{R}$. 

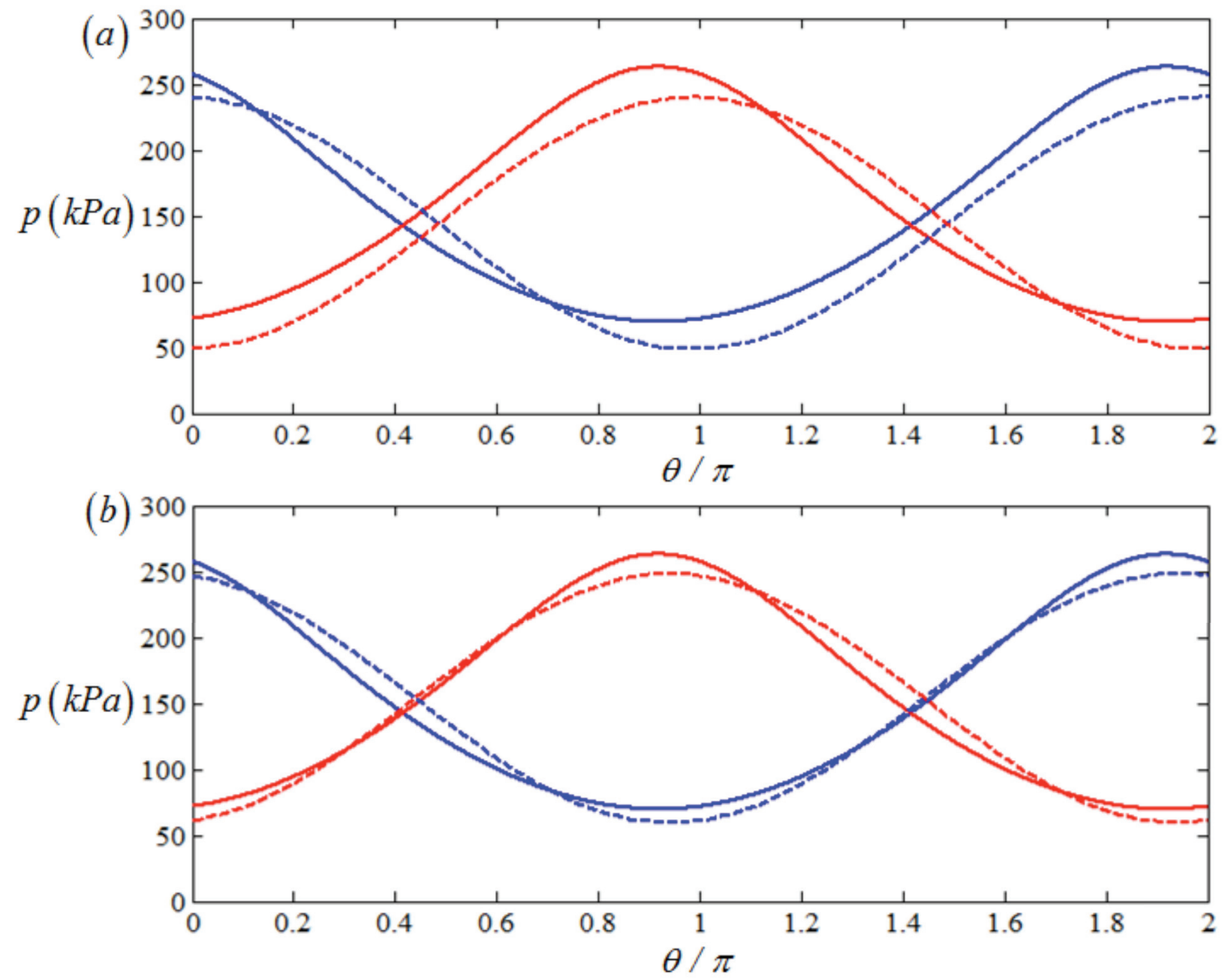

Figure 11. Representative comparison between nonlinear simulation results and solution approximation generated with the single-term harmonic balance method (HBM). (a) Initial guess for mean pressure taken from initial condition in numerical simulation. (b) Initial guess for mean pressure taken by averaging simulated pressure traces. Marginal accuracy is achieved. Key: Simulated $p_{L} ;$ Simulated $p_{R} ;---\operatorname{HBM} p_{L} ;$--- HBM $p_{R}$. 

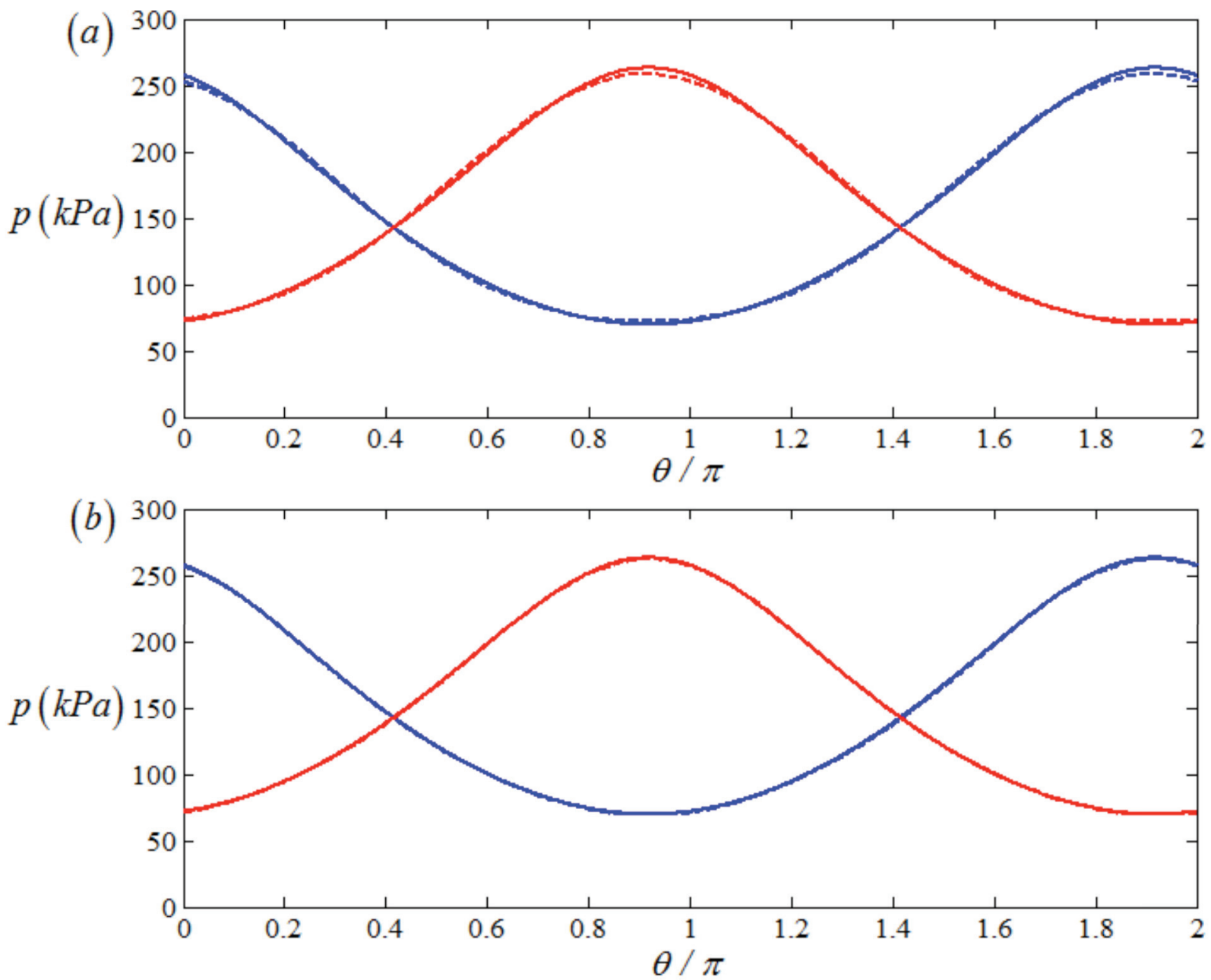

Figure 12. Representative comparison between simulation results and solution approximation generated with the multi-term harmonic balance method using multiple harmonics. ( $a$ ) Two harmonics. Improved accuracy is achieved. (b) Three harmonics. Excellent accuracy is achieved. Key: - Simulated $p_{L} ;$ Simulated $p_{R} ;---$ MHBM $p_{L} ;---\operatorname{MHBM} p_{R}$. 


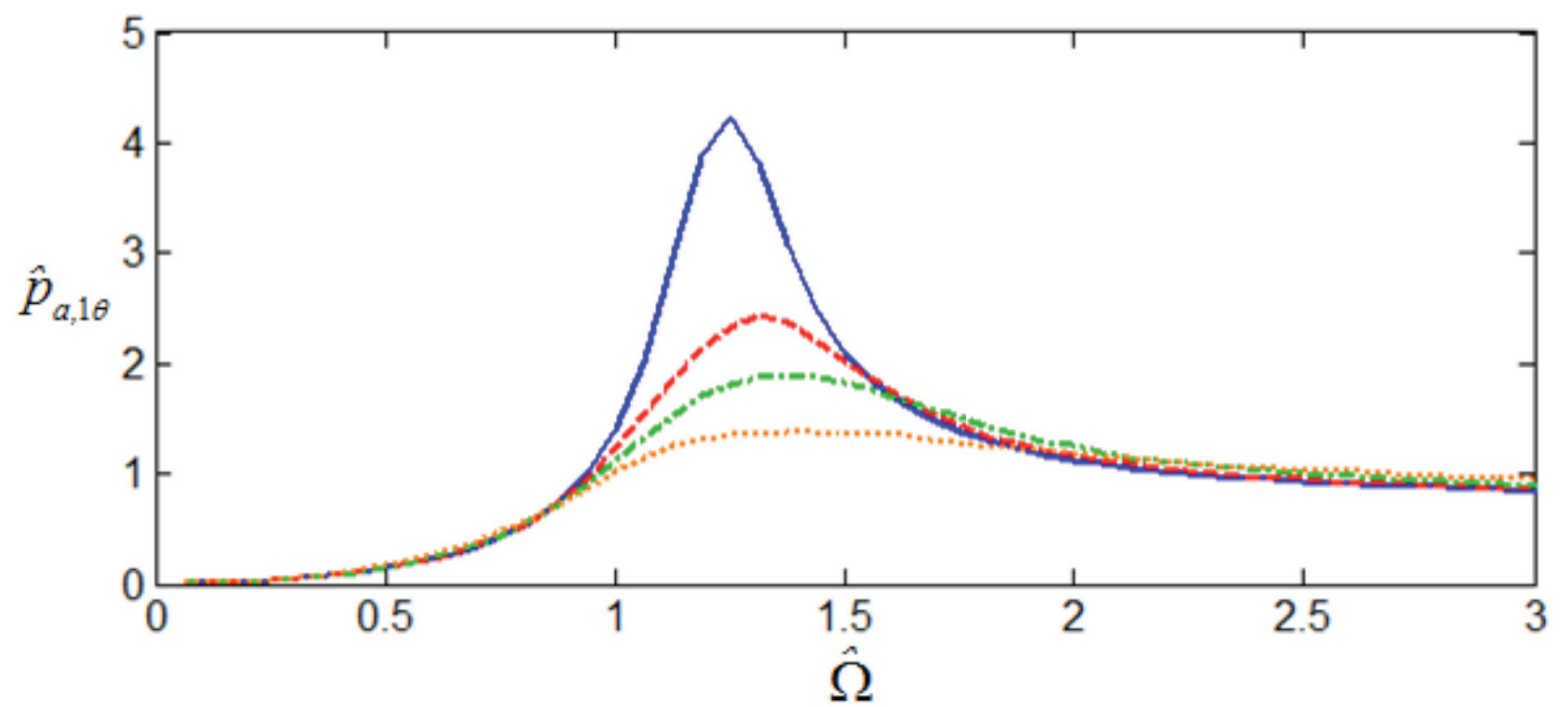

Figure 13. Amplitude of the fundamental (first) pressure harmonic of Model IV. Key: $-x_{a}=$ $0.1 \mathrm{~mm} ;---x_{a}=0.5 \mathrm{~mm} ;-\cdot-x_{a}=1.0 \mathrm{~mm} ; \cdots x_{a}=2.0 \mathrm{~mm}$. 

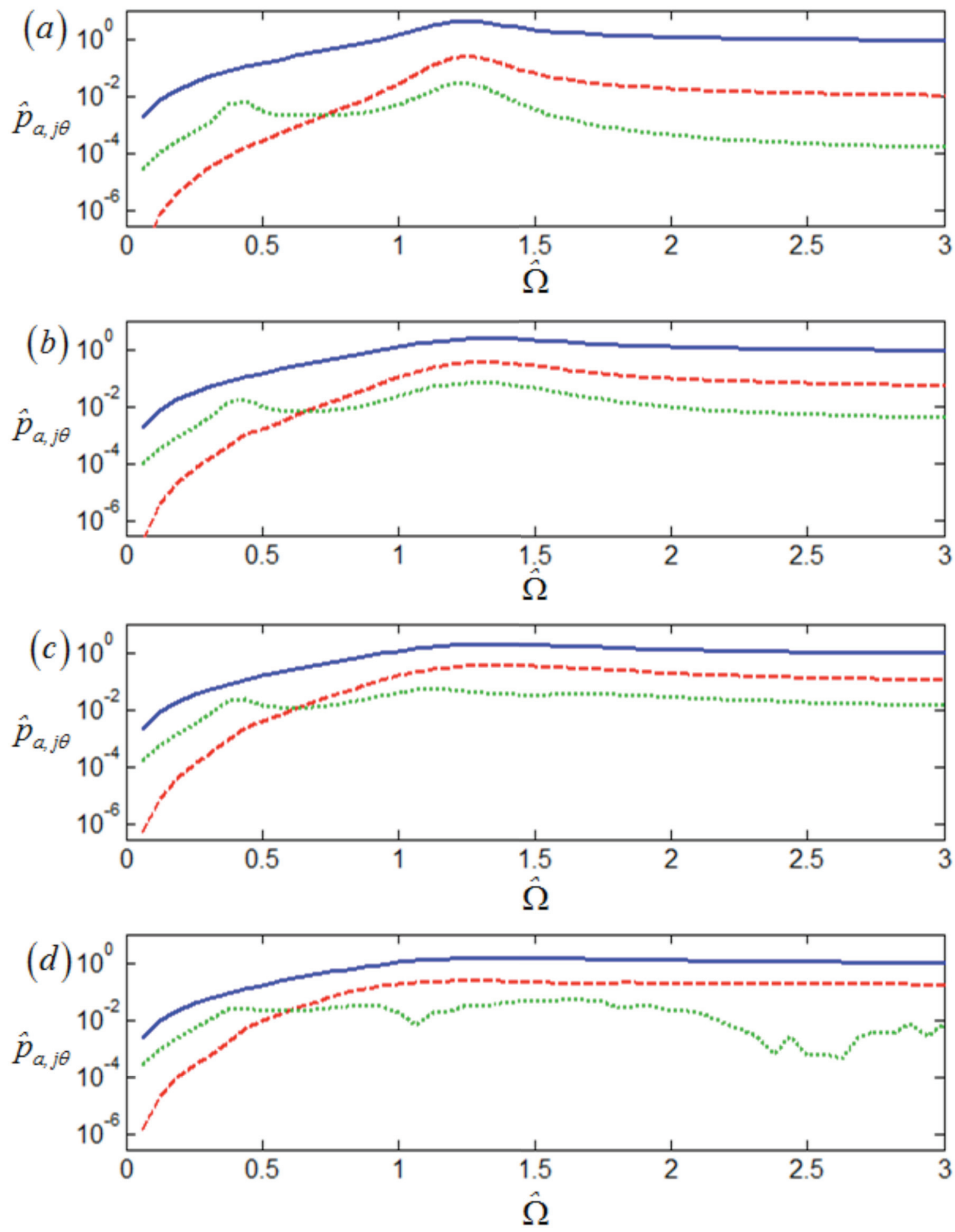

Figure 14. Existence and relative magnitudes of super harmonics as illustrated by harmonic pressure terms in the nonlinear dynamic responses. Sine and cosine coefficients are calculated by the multi-term harmonic balance method, converted to magnitude form, and normalized by 
excitation amplitude. (a) $x_{a}=0.1 \mathrm{~mm},(b) x_{a}=0.5 \mathrm{~mm},(c) x_{a}=1.0 \mathrm{~mm}$, and $(d) x_{a}=2.0 \mathrm{~mm}$.

Key: —— First harmonic; --- second harmonic; ...... third harmonic.
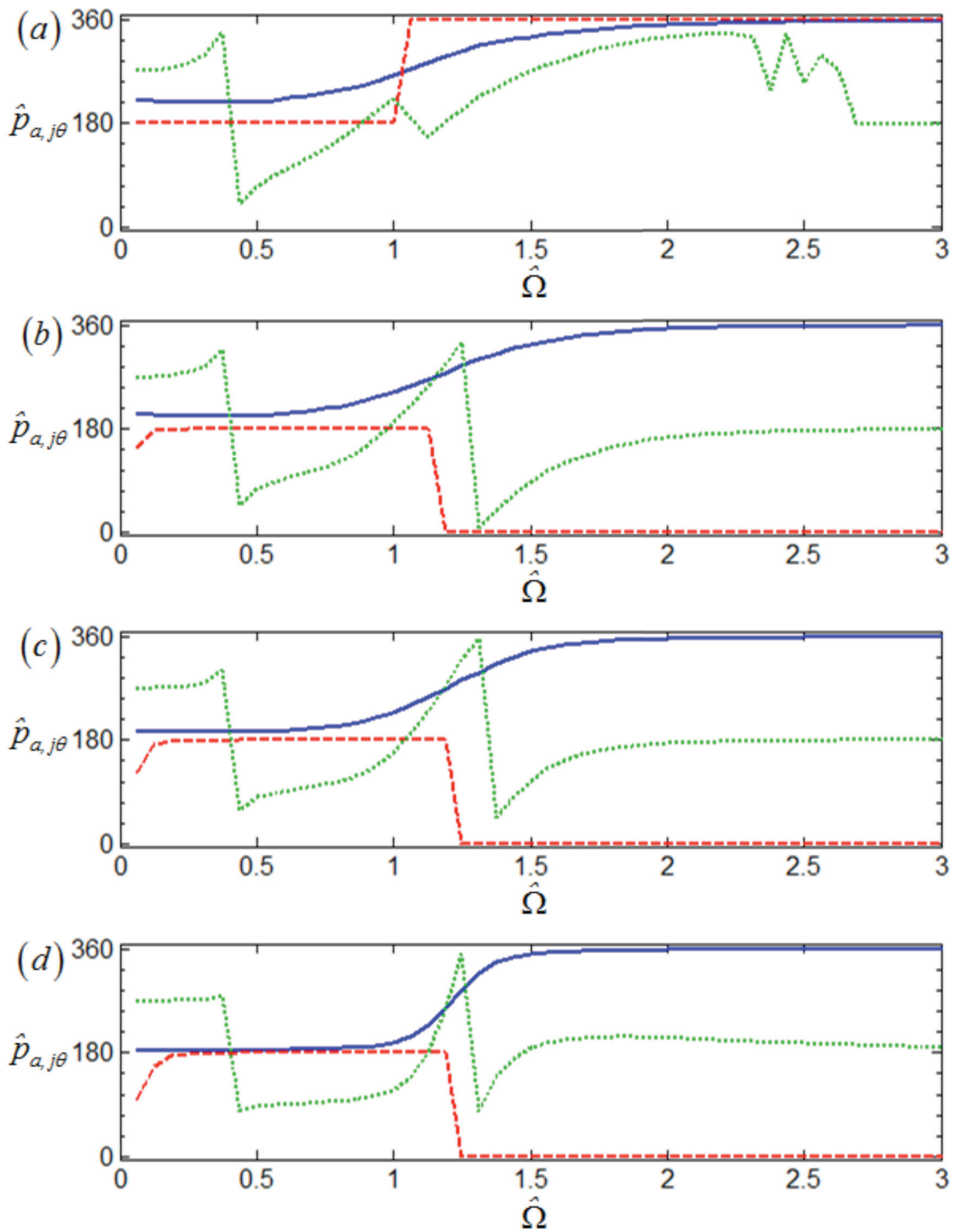
Figure 15. Existence and relative phases of super harmonics as illustrated by harmonic pressure terms in the nonlinear dynamic responses. Sine and cosine coefficients are calculated by the multi-term harmonic balance method and converted to phase form at several excitation amplitude, $(a) x_{a}=0.1 \mathrm{~mm},(b) x_{a}=0.5 \mathrm{~mm},(c) x_{a}=1.0 \mathrm{~mm}$, and $(d) x_{a}=2.0 \mathrm{~mm}$. Key: First harmonic; --- second harmonic; ...... third harmonic.
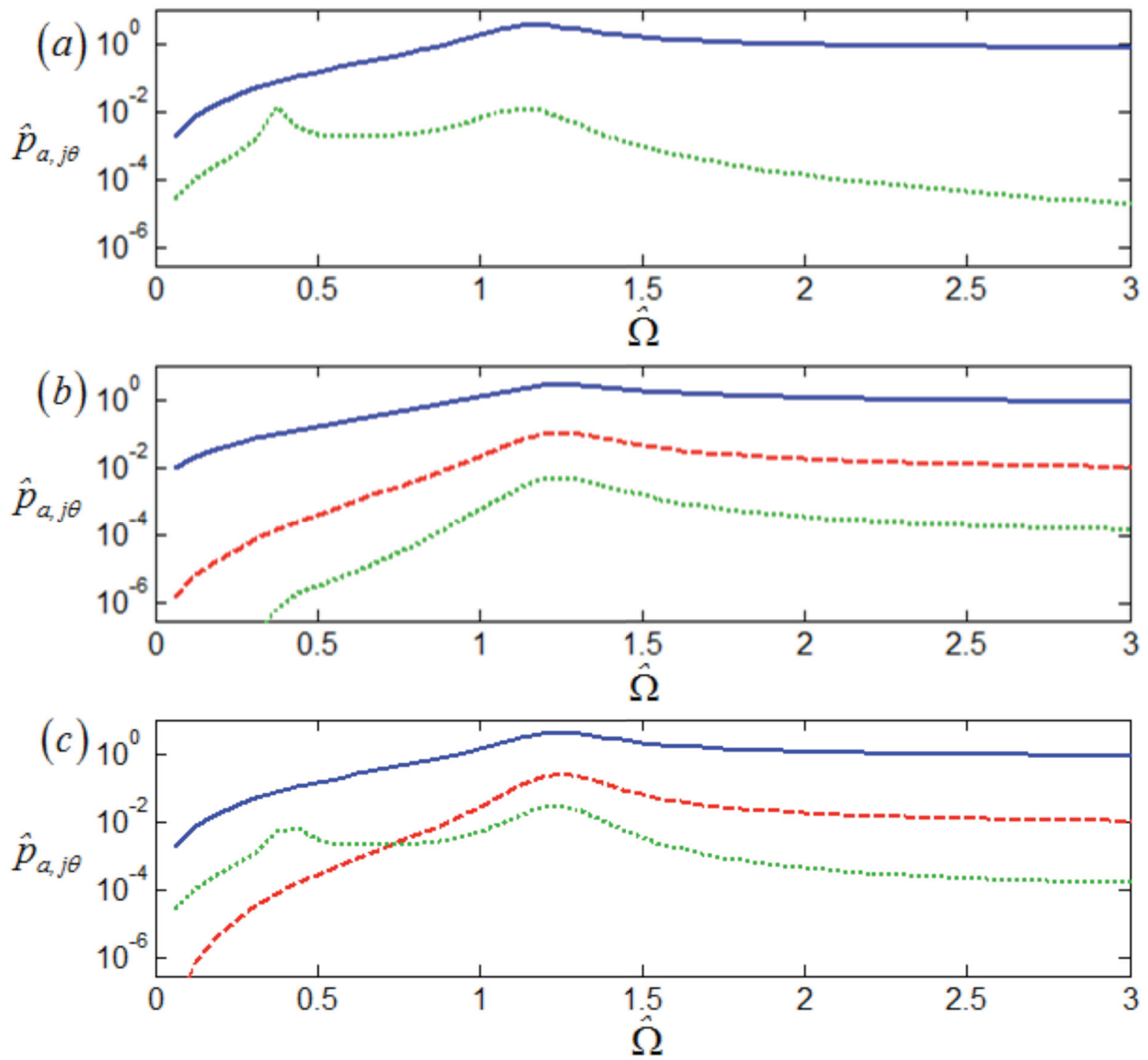
Figure 16. Relative magnitudes of super harmonics as illustrated by harmonic pressure terms in the nonlinear dynamic responses. Sine and cosine coefficients are calculated by the multi-term harmonic balance method, converted to magnitude form, and normalized by excitation amplitude. (a) Model II, (b) Model II, and (c) Model IV. Key: — First harmonic; --- second harmonic; $\cdot \cdots \cdot .$. third harmonic.

Table 1. Effect of displacement excitation amplitude $\left(x_{a}\right)$ on the parameters of quasi-linear model (I). Parameters are normalized by their nominal values with a $0.1 \mathrm{~mm}$ excitation.

\begin{tabular}{ccccccc}
$\begin{array}{c}\text { Amplitude } \\
(\mathrm{mm}, \mathrm{p}-\mathrm{p})\end{array}$ & $\mathrm{I}_{\mathrm{i}}$ & $\mathrm{R}_{\mathrm{i}}$ & $\mathrm{C}$ & $\mathrm{A}$ & $\mathrm{k}_{\mathrm{r}}$ & $\mathrm{c}_{\mathrm{r}}$ \\
\hline 0.1 & 1.00 & 1.00 & 1.00 & 1.00 & 1.00 & 1.00 \\
0.5 & 1.00 & 1.03 & 0.64 & 0.41 & 0.65 & 0.69 \\
1.0 & 1.00 & 1.00 & 0.50 & 0.24 & 0.48 & 0.48 \\
2.0 & 1.00 & 0.90 & 0.40 & 0.13 & 0.34 & 0.14
\end{tabular}

Table 2. Quantification of error, $\varepsilon_{K}$, associated with quasilinear (I) and nonlinear (II, III, and IV) models.

\begin{tabular}{ccrrrrr} 
& & \multicolumn{5}{c}{ Amplitude (mm, p-p) } \\
Model & Description & 0.1 & 0.5 & 1.0 & 2.0 & Avg \\
\hline I & Quasi-Linear & 0.203 & 0.183 & 0.186 & 0.204 & 0.194 \\
II & Only $R(q)$ Nonlinearity & 0.672 & 0.476 & 0.373 & 0.246 & 0.442 \\
III & Only $C(p)$ Nonlinearity & 0.343 & 0.361 & 0.356 & 0.290 & 0.338 \\
IV & Both $R(q)$ and $C(p)$ Nonlinearity & 0.467 & 0.281 & 0.191 & 0.128 & 0.267
\end{tabular}

